\title{
Model-aided radiometric determination of photolysis frequencies in a sunlit atmosphere simulation chamber
}

\author{
B. Bohn ${ }^{1}$ and H. Zilken ${ }^{2}$ \\ ${ }^{1}$ Institut für Chemie und Dynamik der Geosphäre II: Troposphäre, Forschungszentrum Jülich, 52425 Jülich, Germany \\ ${ }^{2}$ Zentralinstitut für Angewandte Mathematik, Forschungszentrum Jülich, 52425 Jülich, Germany
}

Received: 3 September 2004 - Published in Atmos. Chem. Phys. Discuss.: 29 October 2004

Revised: 21 January 2004 - Accepted: 21 January 2004 - Published: 25 January 2005

\begin{abstract}
In this work diurnal and seasonal variations of mean photolysis frequencies for the atmosphere simulation chamber SAPHIR at Forschungszentrum Jülich are calculated. SAPHIR has a complex construction with UV permeable teflon walls allowing natural sunlight to enter the reactor volume. The calculations are based on external measurements of solar spectral actinic flux and a model considering the time-dependent impact of shadows from construction elements as well as the influence of the teflon walls. Overcast and clear-sky conditions are treated in a consistent way and different assumptions concerning diffuse sky radiance distributions are tested. Radiometric measurements inside the chamber are used for an inspection of model predictions. Under overcast conditions we obtain fractions of 0.74 and 0.67 of external values for photolysis frequencies $j\left(\mathrm{NO}_{2}\right)$ $\left(\mathrm{NO}_{2}+h v \rightarrow \mathrm{NO}+\mathrm{O}\left({ }^{3} \mathrm{P}\right)\right)$ and $j\left(\mathrm{O}^{1} \mathrm{D}\right)\left(\mathrm{O}_{3}+h v \rightarrow \mathrm{O}_{2}+\mathrm{O}\left({ }^{1} \mathrm{D}\right)\right)$, respectively. On a clear sky summer day these values are time-dependent within ranges $0.65-0.86$ and $0.60-0.73$, for $j\left(\mathrm{NO}_{2}\right)$ and $j\left(\mathrm{O}^{1} \mathrm{D}\right)$, respectively. A succeeding paper (Bohn et al., 2004) is dealing with an on-road test of the model approach by comparison with photolysis frequencies from chemical actinometry experiments within SAPHIR.
\end{abstract}

\section{Introduction}

Ultraviolet sunlight is driving atmospheric chemistry. Highly reactive species are produced by photolyses of trace gases followed by complex secondary chemistry. For example, photolysis of ozone in the UV-B forms electronically excited $\mathrm{O}\left({ }^{1} \mathrm{D}\right)$ atoms which can react with water vapour producing $\mathrm{OH}$ radicals:

$\mathrm{O}_{3}+h v(\lambda \leq 340 \mathrm{~nm}) \rightarrow \mathrm{O}\left({ }^{1} \mathrm{D}\right)+\mathrm{O}_{2}$

Correspondence to: B. Bohn

(b.bohn@fz-juelich.de)
$\mathrm{O}\left({ }^{1} \mathrm{D}\right)+\mathrm{H}_{2} \mathrm{O} \rightarrow 2 \mathrm{OH}$

$\mathrm{OH}$ radicals react with the majority of trace gases initiating their degradation in the troposphere. In secondary steps peroxy radicals $\left(\mathrm{RO}_{2}\right)$ are produced which oxidise $\mathrm{NO}$ to $\mathrm{NO}_{2}$ in the presence of $\mathrm{NO}_{\mathrm{x}}\left(=\mathrm{NO}+\mathrm{NO}_{2}\right)$. Photolysis of $\mathrm{NO}_{2}$ then leads to a net production of ozone commonly observed in polluted areas:

$$
\begin{aligned}
& \mathrm{RO}_{2}+\mathrm{NO} \rightarrow \mathrm{RO}+\mathrm{NO}_{2} \\
& \mathrm{NO}_{2}+h v(\lambda \leq 420 \mathrm{~nm}) \rightarrow \mathrm{O}\left({ }^{3} \mathrm{P}\right)+\mathrm{NO} \\
& \mathrm{O}\left({ }^{3} \mathrm{P}\right)+\mathrm{O}_{2} \rightarrow \mathrm{O}_{3}
\end{aligned}
$$

Evidently, photolysis processes play a vital role in atmospheric chemistry. They are quantified by first-order rate constants referred to as photolysis frequencies:

$j=\int \sigma(\lambda) \phi(\lambda) F_{\lambda}(\lambda) \mathrm{d} \lambda$

In this equation $F_{\lambda}$ is the solar spectral actinic photon flux, $\sigma$ is the absorption cross section of the absorbing molecule and $\phi$ is the quantum yield of the photo-fragments.

Different photolysis processes are governed by different wavelength dependencies of absorption cross sections of precursor molecules and quantum yields of photo-products. Of course, under natural conditions $F_{\lambda}$ and therefore photolysis frequencies are strongly variable. In field measurements spectral actinic flux $F_{\lambda}$ can be measured, for example, by using double monochromators combined with specially designed detector optics (Müller et al., 1995; Kraus and Hofzumahaus, 1998; Hofzumahaus et al., 1999; Shetter and Müller, 1999). A measurement of $F_{\lambda}$ allows determining any photolysis frequency if the parameters $\sigma$ and $\phi$ are known. 


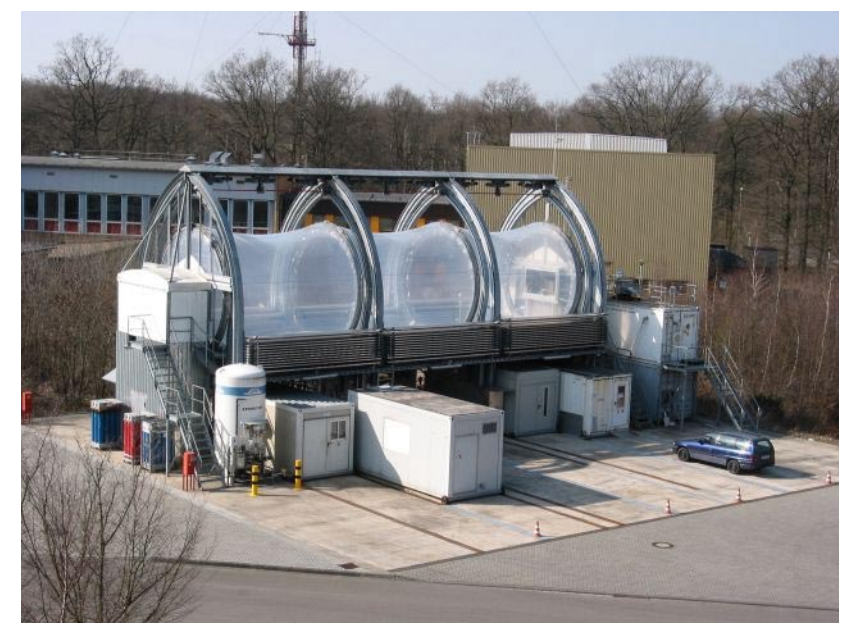

Fig. 1. The atmosphere simulation chamber SAPHIR with opened roof. The photograph was taken from the site of the external spectroradiometer measurements.

The atmosphere simulation chamber SAPHIR at Forschungszentrum Jülich $\left(50.91^{\circ} \mathrm{N}, 6.41^{\circ} \mathrm{E}\right.$, Fig. 1) was designed to study tropospheric chemistry under ambient conditions with respect to temperature, pressure and UV-radiation. Natural sunlight is used as a light source entering the chamber through UV-permeable teflon walls. The concentrations of trace gases are comparable to ambient levels but, in contrast to field experiments, chemical composition is controlled and not affected by transport processes. This separation of transport and chemistry allows a more precise experimental study of tropospheric chemistry by applying the usual range of techniques established in field experiments including radical measurements.

However, with respect to spectral actinic flux and photolysis frequency measurements the situation is less favourable. While under tropospheric conditions, photolysis frequencies are variable at a given location as a result of diurnal and seasonal variations there is usually little small-scale spatial variability. Under field conditions, a photolysis frequency measurement is therefore considered representative for a larger area, at least with regard to short-lived species like $\mathrm{HO}_{\mathrm{x}}$ $\left(=\mathrm{OH}+\mathrm{HO}_{2}\right)$. Therefore, although there may be exceptions under broken cloud conditions, steady-state approximations are a reasonable assumption for $\mathrm{HO}_{\mathrm{x}}$ field-data analyses by using photolysis frequencies measured close to the $\mathrm{HO}_{\mathrm{x}}$ measurement site and neglecting transport. However, this approach does not apply for SAPHIR. The radiation field within the inner reactor is complex because it is influenced by shadows cast by construction elements as well as reflections and scattering at chamber walls. Chemical data analysis (for most purposes) needs mean photolysis frequencies for the reactor as a whole but radiometric measurements within the reactor are only feasible at few selected points. As a consequence, these measurements are not appropriate.

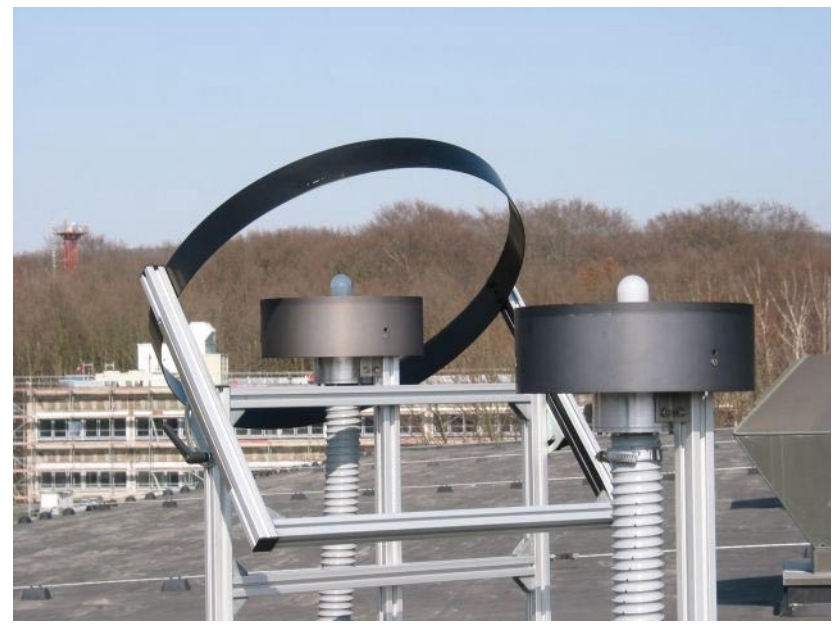

Fig. 2. Actinic flux detector heads of the spectroradiometer with shadow ring obstructing direct sunlight.

In this work we present an approach to derive mean photolysis frequencies for SAPHIR based on outside measurements of spectral actinic flux. This approach was developed after a number of experiments with radiometric sensors positioned inside and outside of SAPHIR. It turned out that caused by local effects (mainly shadows) the conversion to mean photolysis frequencies is less complicated if it is based on measurements outside the chamber rather than inside. The conversion concept is composed of (i) a distinction between direct sunlight and diffuse sky radiation, (ii) a model predicting relative time dependencies for these light sources and (iii) an absolute calibration using the whole chamber as a chemical actinometer. The last point is described in a separate paper (Bohn et al., 2004) also addressing the influence of local differences of actinic flux within the chamber.

It should be noted that despite the difficulties associated with the use of natural sunlight artificial light sources are not considered an alternative. In SAPHIR we want to study tropospheric chemistry by monitoring key species like $\mathrm{HO}_{\mathrm{x}}$, $\mathrm{RO}_{\mathrm{x}}$ and $\mathrm{NO}_{\mathrm{x}}$ in environments of varying complexity to understand the dynamics of the chemical system as a whole under conditions close to the natural atmosphere. Although the advantage of an artificial light source would be its constancy and availability at any time, it is difficult to simulate the spectral properties and intensity of natural sunlight by artificial light sources. For example it is hard to reproduce the typical ratio of photolysis frequencies in the troposphere, e.g. the ratio $j\left(\mathrm{O}^{1} \mathrm{D}\right) / j\left(\mathrm{NO}_{2}\right)$ which directly affects the relationship between the transient species mentioned above. Moreover, there is a variety of atmospheric photolysis processes not accounted for quantitatively because absorption cross sections and quantum yields are poorly known. Using natural sunlight at least makes sure that the net effect of these unaccounted processes is comparable to ambient conditions. 


\section{Experimental}

\subsection{Radiometric measurements}

A co-channel spectroradiometer (Bentham 300) was used to measure the $2 \pi$ sr solar spectral actinic flux on the roof of a building close to SAPHIR (distance $70 \mathrm{~m}, 12 \mathrm{~m}$ above chamber ground). Spectra were taken in the range $280-420 \mathrm{~nm}$ in most cases with a spectral resolution of $1 \mathrm{~nm}$ which takes about $2 \mathrm{~min}$. Absolute calibration of the spectroradiometer was made using a $1000 \mathrm{~W}$ irradiance standard (BN-9101, Gigahertz-Optik, PTB traceable) and $45 \mathrm{~W}$ secondary standards (Optronic Laboratories) for regular checks. From the spectra, photolysis frequencies were calculated using literature data on absorption cross sections and quantum yields for the process under consideration (Eq. 6). Taking into account the uncertainties of the calibration standard and the optical characterisation of the detector heads, the accuracy of the method is of the order 5-7\% in a wavelength range 300$400 \mathrm{~nm}$, not considering uncertainties of the molecular data $\sigma$ and $\phi$. More details on the spectroradiometer, the detector heads and the determination of photolysis frequencies are given by Hofzumahaus et al. (1999).

A shadow ring obstructing direct sunlight was used with one of the detector heads (Fig. 2, left). The ring has a diameter of $600 \mathrm{~mm}$ and a width of $60 \mathrm{~mm}$. The ring plane is tilted by an angle of $39^{\circ}\left(=90^{\circ}-\right.$ latitude $)$ with respect to the horizontal in south-north direction. At equinox the detector head is positioned at the centre of the ring plane. In the course of a year the ring has to be shifted up and down along an axis perpendicular to the ring plane. Typically, adjustments have to be made every four days. The actinic flux measured with the shaded detector head is corrected with respect to the solid angle obstructed by the ring in the upper hemisphere. The corresponding geometrical correction factor was derived analytically. It varies between 1.05 and 1.13 at winter and summer solstice, respectively. This correction is assuming an isotropic radiance distribution of diffuse sky radiation which does not apply for natural conditions. As a consequence, other correction factors will be derived in Sect. 3.5. In order to test these ring corrections experimentally, spot check measurements were made where direct sunlight was blocked manually also from the unshaded detector (Fig. 2, right) by a disk obstructing an about $6^{\circ}$ area around the sun. This area corresponds to the minimum obstruction by the shadow ring in direction perpendicular to the ring plane. Accordingly, diffuse sky radiation coming from a $6^{\circ}$ area around the sun is considered direct sunlight which is sufficiently precise for the intention of this work. A different approach to measure the sky radiation blocked by the ring is described in the literature: the ring is displaced just off the sun and the resulting flux is subtracted from the total flux. This method is specially suitable for fast measurements where it can be applied continuously and automatically with a single detector using a rotating shadow band (Harrison et al., 1994).
Some spectroradiometer measurements were made within the simulation chamber to determine the ratios of up- and down-welling actinic flux. Moreover, measurements with two spectroradiometers operating simultaneously inside and outside the chamber were made to investigate the spectral influence of the chamber walls. The angle dependent spectral transmittance of the chamber wall material was also determined in laboratory experiments using the spectroradiometer.

\subsection{Simulation chamber}

The simulation chamber SAPHIR consists of an almost cylindrical, double-wall teflon tube held in a steel frame (Fig. 1). The chamber is aligned with its long axis in northsouth direction. The inner tube $(r=2.5 \mathrm{~m}, l=18.4 \mathrm{~m})$ is used as a reactor for simulation experiments $\left(V=270 \mathrm{~m}^{3}\right)$. The gap between the inner and the outer tube is about $0.1 \mathrm{~m}$. The inner tube consists of FEP film with a thickness of $125 \mu \mathrm{m}$ except from the ground $\left(52 \mathrm{~m}^{2}\right)$ made of $500 \mu \mathrm{m}$ FEP film. The outer tube consists of $250 \mu \mathrm{m}$ FEP material. Teflon FEP (DuPont) is a co-polymer of fluorinated ethene and propene. It has been selected because it is chemically inert and UV permeable allowing sunlight to enter the chamber. Moreover, FEP is remarkably stable in terms of weathering. According to the manufacturer there was no measurable change of the material after 20 years of outdoor exposure in Florida. A reduction of transmittance by staining is prevented by regular cleaning and samples of the material are kept for laboratory checks. In case of an exchange of material, e.g. caused by damage, any change of properties can be ascertained using these samples.

The reactor can be covered by a movable, opaque roof construction within about $1 \mathrm{~min}$ and vice versa. Upon closing the roof, the signal of a $j\left(\mathrm{NO}_{2}\right)$ filterradiometer inside the chamber (measuring integrated actinic flux in the UV-A) decreases by at least a factor of $10^{3}$. More details concerning the instrumentation and performance of SAPHIR are given by Rohrer et al. (2004) and Bohn et al. (2004).

\section{Model calculations}

Light coming from different directions is entering the chamber with different efficiency because SAPHIR has no hemispheric symmetry. This results in time-dependent effects caused by opaque construction elements (shadows) and the FEP walls (angle- and wavelength dependent transmission).

The general idea already outlined in the introduction is to describe the mean spectral actinic flux inside of SAPHIR in terms of the external fluxes $F_{\lambda}^{\text {diffuse }}$ and $F_{\lambda}^{\text {direct: }}$

$$
\begin{aligned}
& F_{\lambda}^{\text {diffuse }}(\lambda)=F_{\lambda}^{\text {ring }}(\lambda) f_{\text {ring }} \\
& F_{\lambda}^{\text {direct }}(\lambda)=F_{\lambda}^{\text {total }}(\lambda)-F_{\lambda}^{\text {ring }}(\lambda) f_{\text {ring }}
\end{aligned}
$$


In these equations $F_{\lambda}^{\text {ring }}$ and $F_{\lambda}^{\text {total }}$ are the spectral actinic fluxes measured outside with and without shadow ring, respectively. $f_{\text {ring }}$ is the shadow ring correction factor.

Mean chamber fluxes $F_{\lambda}^{\mathrm{c}}$ are obtained by applying weighting factors $f_{\text {direct }}$ and $f_{\text {diffuse }}$ to the two components, as well as an absolute scaling factor $h^{\mathrm{c}}$.

$$
\begin{aligned}
F_{\lambda}^{\mathrm{c}}(\lambda, t)=h^{\mathrm{c}} & \left\{F_{\lambda}^{\text {direct }}(\lambda) f_{\text {direct }}(t, \lambda)\right. \\
& \left.+F_{\lambda}^{\text {diffuse }}(\lambda) f_{\text {diffuse }}(t, \lambda)\right\}
\end{aligned}
$$

The time- and wavelength-dependent weighting factors will be derived from model calculations in this work including some experimental input, while the constant scaling factor $h^{\mathrm{c}}$ is determined experimentally in actinometric experiments (Bohn et al., 2004). However, $4 \pi$ spectroradiometric measurements of actinic flux within the chamber will be used in this work to derive a preliminary $h^{\mathrm{c}}$.

To allow a distinction between effects caused by shadows and the FEP walls, two direction weighting functions are defined by the following integrals:

$$
\begin{aligned}
& f^{\mathrm{V}}(\vartheta, \varphi)=\frac{1}{V^{\mathrm{c}}} \int_{V^{\mathrm{c}}} s(\boldsymbol{r}, \vartheta, \varphi) d V \\
& f^{\mathrm{T}}(\vartheta, \varphi, \lambda)=\frac{1}{V^{\mathrm{c}}} \int_{V^{\mathrm{c}}} s(\boldsymbol{r}, \vartheta, \varphi) T(\boldsymbol{r}, \vartheta, \varphi, \lambda) d V
\end{aligned}
$$

$V^{\mathrm{c}}$ is the inner volume of the chamber. $s$ is a location dependent function denoting whether or not a location $\boldsymbol{r}$ is receiving light from a direction in the sky described by its zenithand azimuth angles, $\vartheta$ and $\varphi$, respectively, i.e. $s=\{0,1\}$. $T$ is the corresponding transmittance of the FEP walls dependent on wavelength and angle of incidence. Thus, $f^{\mathrm{V}}$ is the illuminated volume fraction neglecting the influence of the FEP walls while $f^{\mathrm{T}}$ considers the combined effects of shadows and FEP wall transmittance. Only the upper hemisphere is considered in this treatment, i.e. $\vartheta \leq 90^{\circ}$. The lower hemisphere is neglected because of low ground albedo of the surrounding area and for geometrical reasons (opaque chamber ground).

It should be noted that this model is neglecting scattering processes at the FEP walls and reflections within the chamber (e.g. at the chamber ground). Scattering processes are addressed indirectly in Sects. 3.2 and 4.3. Reflections within the chamber are expected to lead to an amplification of actinic flux which will be assigned to the scaling factor $h^{\mathrm{c}}$ of Eq. (9), i.e. $h^{\mathrm{c}}>1$ is expected (Sect. 4.4).

The integrals of Eqs. (10) and (11) are approximated by averaging over a sufficient number of uniformly distributed locations within the reactor volume:

$$
\begin{aligned}
& f^{\mathrm{V}}(\vartheta, \varphi) \approx \frac{1}{N} \sum_{n=0}^{N} s\left(\boldsymbol{r}_{n}, \vartheta, \varphi\right) \\
& f^{\mathrm{T}}(\vartheta, \varphi, \lambda) \approx \frac{1}{N} \sum_{n=0}^{N} s\left(\boldsymbol{r}_{n}, \vartheta, \varphi\right) T\left(\boldsymbol{r}_{n}, \vartheta, \varphi, \lambda\right)
\end{aligned}
$$

Weighting factors $f_{\text {direct, } \mathrm{V}}$ and $f_{\text {direct,T }}$ for direct sunlight in terms of Eq. (9) are derived from the direction weighting functions by inserting the corresponding solar zenith- and azimuth angles at time $t, \vartheta_{\circ}$ and $\varphi_{\circ}$, respectively.

$$
\begin{aligned}
& f_{\text {direct, } \mathrm{V}}(t)=f^{\mathrm{V}}\left(\vartheta_{\circ}, \varphi_{\circ}\right) \\
& f_{\text {direct, } \mathrm{T}}(t, \lambda)=f^{\mathrm{T}}\left(\vartheta_{\circ}, \varphi_{\circ}, \lambda\right)
\end{aligned}
$$

For diffuse sky radiation the corresponding weighting factors are derived from $f^{\mathrm{V}}$ and $f^{\mathrm{T}}$ by multiplication with the $2 \pi$ sr-normalised radiance $L$ and integration of the upper hemisphere.

$$
\begin{aligned}
f_{\text {diffuse }, \mathrm{V}}(t)=\int_{0}^{2 \pi} & \int_{0}^{\pi / 2} f^{\mathrm{V}}(\vartheta, \varphi) \\
& \times L(\vartheta, \varphi, t) \sin (\vartheta) d \vartheta d \varphi \\
f_{\text {diffuse }, \mathrm{T}}(t, \lambda)= & \int_{0}^{2 \pi} \int_{0}^{\pi / 2} f^{\mathrm{T}}(\vartheta, \varphi, \lambda) \\
& \times L(\vartheta, \varphi, t) \sin (\vartheta) d \vartheta d \varphi
\end{aligned}
$$

Any wavelength dependence of radiance $L$ is neglected here (see Sect. 3.3). The integrations of Eqs. (16) and (17) were also approximated for numerical calculation, e.g. in the case of $f_{\text {diffuse, } \mathrm{T}}$ :

$f_{\text {diffuse }, \mathrm{T}}(t, \lambda) \approx \frac{\sum_{p=0}^{P} \sum_{q=0}^{Q} f^{\mathrm{T}}\left(\vartheta_{p}, \varphi_{q}, \lambda\right) L\left(\vartheta_{p}, \varphi_{q}, t\right)}{\sum_{p=0}^{P} \sum_{q=0}^{Q} L\left(\vartheta_{p}, \varphi_{q}, t\right)}$

with $\quad \vartheta_{p} / \operatorname{deg}=p 90 / P, \quad Q=4 P \sin \left(\vartheta_{p}\right) \quad$ and $\varphi_{q} / \mathrm{deg}=q 360 / Q$. The integer $Q$ is obtained by rounding the term $4 P \sin \left(\vartheta_{p}\right)$. This calculation provides a homogeneous scan of the upper hemisphere with respect to solid angle with adjustable resolution. For $f_{\text {diffuse }}^{\mathrm{V}}$ a corresponding expression applies by inserting $f^{\mathrm{V}}$ instead of $f^{\mathrm{T}}$ in Eq. (18).

\subsection{Steel frame, $s$ and $f^{\mathrm{V}}$}

A CAD model of the chamber steel frame was obtained from the construction company (Plantec GmbH, Bremen) where the surfaces of all solid elements are described by triangles. This model was extended by objects mounted at the steel frame, e.g. sealing bars holding the FEP film and guideways of roller shutters. The original resolution was then downgraded reducing the number of triangles from 32000 to 4400 . This reduction was made to save computing time and affects curved parts of the construction approximated by straight elements. The description is still reasonable, as is evident from Fig. 3.

A programme was developed which can process the triangle data to obtain the function $s$ of Eqs. (10)-(13) for any location $\boldsymbol{r}$. Basically it is checked whether or not any of the 


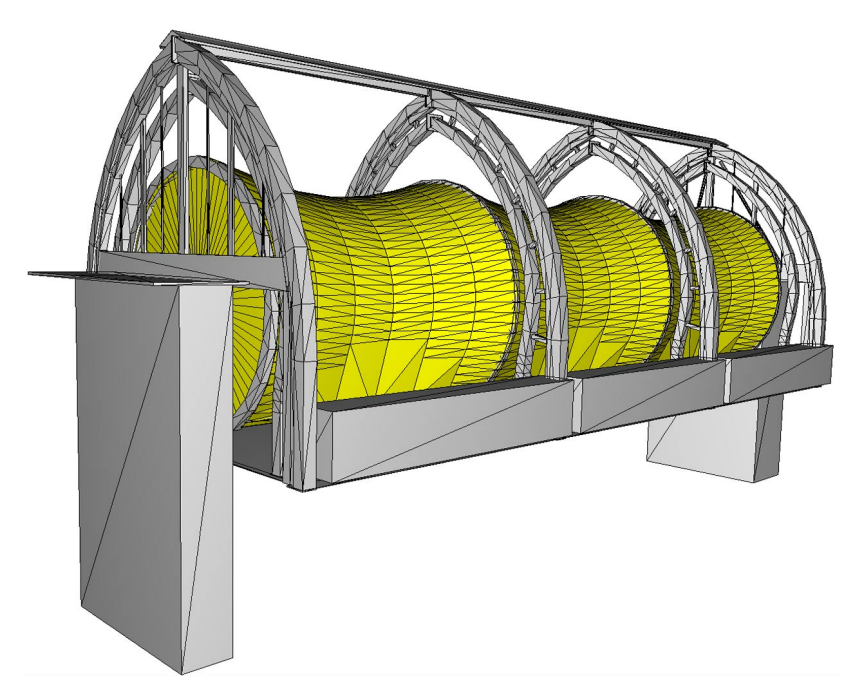

Fig. 3. Model of the SAPHIR frame construction (grey) and FEP tubes (yellow) approximated by 4400 and 1970 triangles, respectively.

triangles are blocking light from direction $\vartheta, \varphi$. The fraction $f^{\mathrm{V}}$ of the illuminated reactor volume was obtained by scanning the reactor volume and averaging the resulting $s$ (Eq. 12). Tests showed that $f^{\mathrm{V}}$ became constant at a spatial resolution of $0.1 \mathrm{~m}$ or better. The bulk of calculations was therefore made with this resolution where each location $r$ represents $10^{-3} \mathrm{~m}^{3}$ of air in the chamber $(N \approx 270000)$.

For a total of about $1400 \vartheta, \varphi$-combinations in the range $\vartheta=0-90^{\circ}$ and $\varphi=90-180^{\circ}$ the corresponding calculations were made and the $f^{\mathrm{V}}$ as well as the arrays of $s$ were saved. These calculations we the most time-consuming step of the computation and took about two days on a personal computer (Pentium 4). The limited range of azimuth angles was selected for symmetry reasons. The $\vartheta, \varphi$-combinations were chosen to obtain an approximately homogeneous scan of the upper hemisphere. The array of $f^{\mathrm{V}}(\vartheta, \varphi)$ results was then parameterised by a function returning the illuminated volume fraction upon input of zenith- and azimuth angle. In Fig. 4 the data are shown in a contour plot projection of the hemisphere. The results are strongly variable. They range from 0.24 in few directions at $\varphi=0^{\circ}\left(180^{\circ}\right)$ and very large zenith angles, to 0.96 at $\varphi=90^{\circ}\left(270^{\circ}\right)$ at moderate zenith angles.

\subsection{FEP walls, $T$ and $f^{\mathrm{T}}$}

\subsubsection{Angles of incidence}

Light entering the inner volume of SAPHIR is influenced by the FEP walls. Similar to the steel frame, a triangle-based model of the FEP walls of the chamber was constructed. A total of 1970 triangles was used for each tube to describe the partly curved areas (see Fig. 3). A programme was developed which for a selected location $\boldsymbol{r}$ calculates the angle of incidence $\alpha$ with respect to the FEP walls for light from

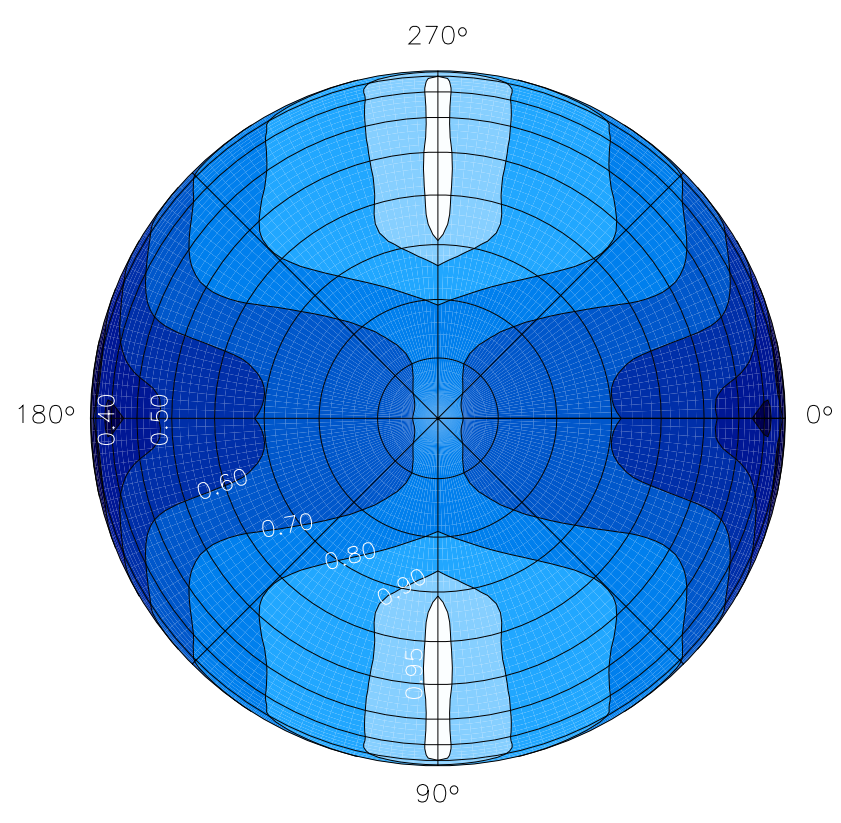

Fig. 4. Contour plot of direction weighting function $f^{\mathrm{V}}$ (illuminated volume fraction) as a function of $\vartheta$ and $\varphi$ (orthographic projection looking from the zenith). Azimuth angles $\varphi$ are indicated $\left(0^{\circ}=\right.$ north, $180^{\circ}=$ south $)$.

any direction $\vartheta, \varphi$. For the same $\vartheta, \varphi$-combinations and locations within the reactor as in the previous section, the $\alpha$ were calculated and saved in output files. Calculations were only made for the outer wall because the narrow interfacial gap produces negligible differences.

\subsubsection{FEP transmittance}

Furthermore, transmittances corresponding to the angles of incidence for each location $\boldsymbol{r}$ are needed to calculate the $f^{\mathrm{T}}$ according to Eq. (13). In general the transmittance $T$ is expected to depend on wavelength, angle of incidence and thickness of the material. Angle dependent transmittance measurements were made with FEP films in the laboratory using the spectroradiometer. These measurements accounted for light transmitted in a direction similar to the direction of incidence. The corresponding transmittance is denoted $T_{\mathrm{d}}$. Scattered light transmitted in other directions could not be measured with the available equipment. However, literature data show that the corresponding transmittance $\left(T_{\mathrm{S}}\right)$ is considerable for FEP (Wallner, 2000; Wallner et al., 2002) in particular with decreasing wavelength. Our measurements of $T_{\mathrm{d}}$ therefore underestimate total, hemispheric transmittance $\left(T_{\mathrm{h}}\right)$. Although scattering processes are not considered explicitly in the model (Eq. 11), the use of $T_{\mathrm{h}}$ is presumed to be more appropriate for the simulation chamber because also the scattered light is entering the chamber. Yet, angle dependent measurements of $T_{\mathrm{h}}$ are not available in the literature making the following considerations necessary. 

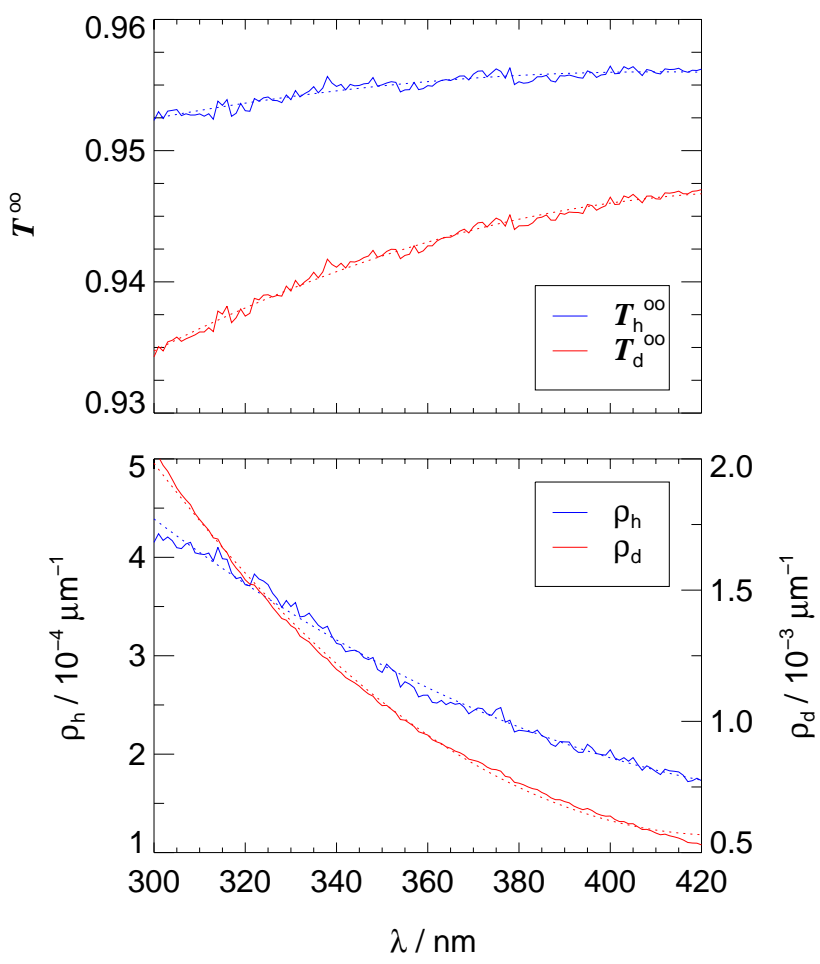

Fig. 5. Parameters fitted to transmittance data from the literature (Wallner, 2000) describing hemispheric and direct transmittance of FEP film at $\alpha=0$ as a function of thickness according to Eq. 20 (full lines) and fitted second-order polynomials (dotted lines). Upper panel: Hemispheric and direct transmittance extrapolated to $d=0$. Lower panel: optical densities caused by bulk effects (mainly scattering).

Wallner (2000) measured $T_{\mathrm{h}}$ and $T_{\mathrm{s}}$ of FEP film at different thicknesses in the range $d=12-125 \mu \mathrm{m}$ at $\alpha=0$. The measurements were made using a spectral photometer and an integrating sphere, i.e. light was detected with equal sensitivity independent of direction of transmission. Consequently also $T_{\mathrm{d}}$ can be determined:

$T_{\mathrm{d}}=T_{\mathrm{h}}-T_{\mathrm{s}}$

In this work we applied exponential fits as a function of $d$ to the $T_{\mathrm{h}}$ and $T_{\mathrm{d}}$ measured by Wallner (2000) for each wavelength in the range $300-420 \mathrm{~nm}$ :

$T_{\mathrm{h}, \mathrm{d}}^{0}(\lambda, d)=T_{\mathrm{h}, \mathrm{d}}^{00}(\lambda) \exp \left\{-\rho_{\mathrm{h}, \mathrm{d}}(\lambda) d\right\}$

The upper index 0 denotes an angle of incidence $\alpha=0$, the index 00 means $\alpha=0$ and $d=0$. The lower index h, d denotes either hemispheric or direct. As is shown in Fig. 5, the fitted parameters $T_{\mathrm{h}}^{00}, T_{\mathrm{d}}^{00}, \rho_{\mathrm{h}}$ and $\rho_{\mathrm{d}}$ exhibit a smooth dependence on wavelength. Therefore, we use the fitted secondorder polynomials also shown in Fig. 5 to describe the wavelength dependence of the parameters in the following. The parameters $T_{\mathrm{h}}^{00}$ and $T_{\mathrm{d}}^{00}$ are somewhat lower than expected for pure specular transmission calculated for a refractive in$\operatorname{dex} n=1.35$ (Fresnel equations, $T_{\mathrm{F}}^{0}=0.956$ ). This can be ex-
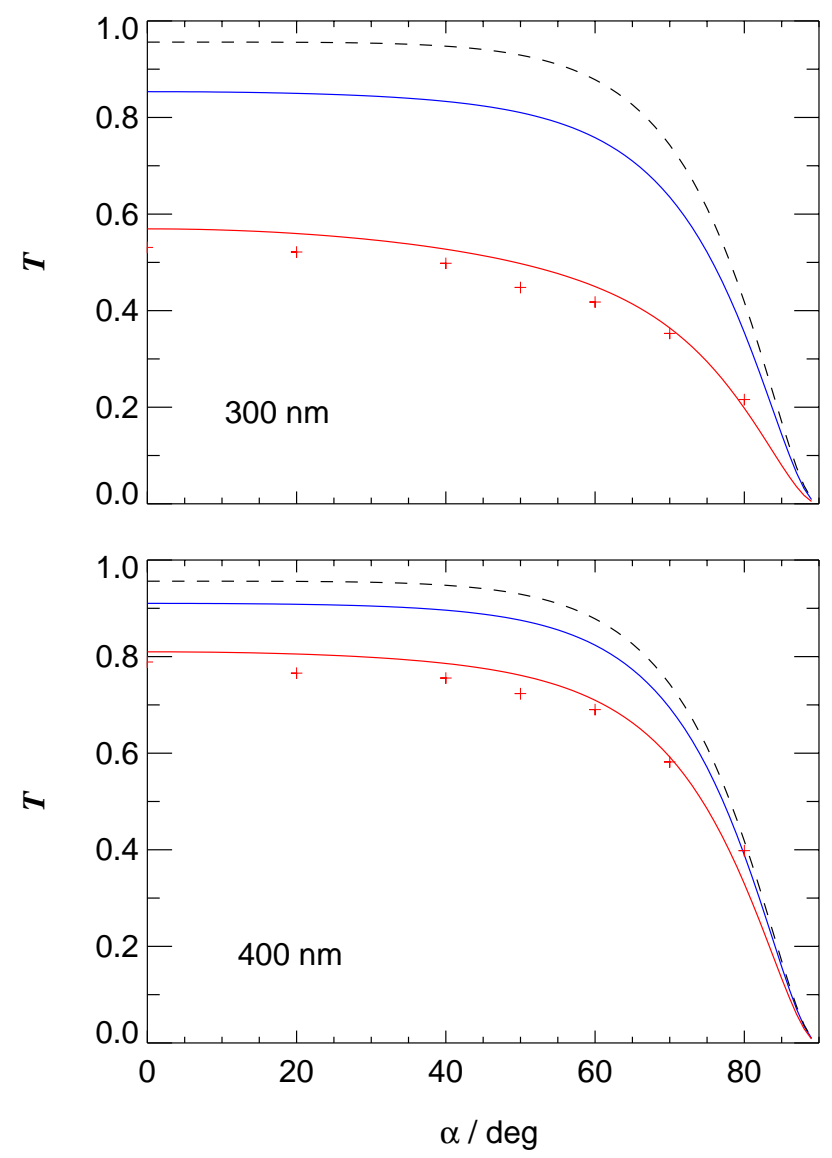

Fig. 6. Angle of incidence dependencies of FEP transmittance $T_{\mathrm{d}}$ (full lines, red) and $T_{\mathrm{h}}$ (full lines, blue) according to Eq. (21) for a $250 \mu \mathrm{m}$ FEP film. Upper panel: $\lambda=300 \mathrm{~nm}$, lower panel: $\lambda=400 \mathrm{~nm}$. Crosses indicate experimental data for the FEP material used in the construction of SAPHIR. The dashed black line shows $T_{\mathrm{F}}$ for comparison (no bulk effects, see text).

plained by scattering losses occurring at the surface of the film. The effect is expectedly more pronounced for $T_{\mathrm{d}}$. Information on the morphology of the film surface can be found elsewhere (Teichert et al., 2002). The optical densities $\rho_{\mathrm{h}}$ and $\rho_{\mathrm{d}}$ increase towards smaller wavelengths which can be explained by increasing scattering losses in the bulk of the film also affecting $T_{\mathrm{d}}$ more strongly.

For the $\alpha$-dependence of the transmittances $T_{\mathrm{h}}$ and $T_{\mathrm{d}}$ of FEP film of any thickness the following relationship is assumed:

$$
\begin{aligned}
T_{\mathrm{h}, \mathrm{d}}(\lambda, \alpha, d) & =T_{\mathrm{h}, \mathrm{d}}^{00}(\lambda) T_{\mathrm{F}}(\alpha) / T_{\mathrm{F}}^{0} \\
& \times \exp \left\{-\rho_{\mathrm{h}, \mathrm{d}}(\lambda) d / \cos (\beta)\right\}
\end{aligned}
$$

Here $\beta$ is the angle of transmission and $T_{\mathrm{F}}$ is the angle dependent specular transmittance neglecting any bulk effects (Fresnel equations). No angle correction for diffusively transmitted light is made which is justified in the case of isotropic scattering. 


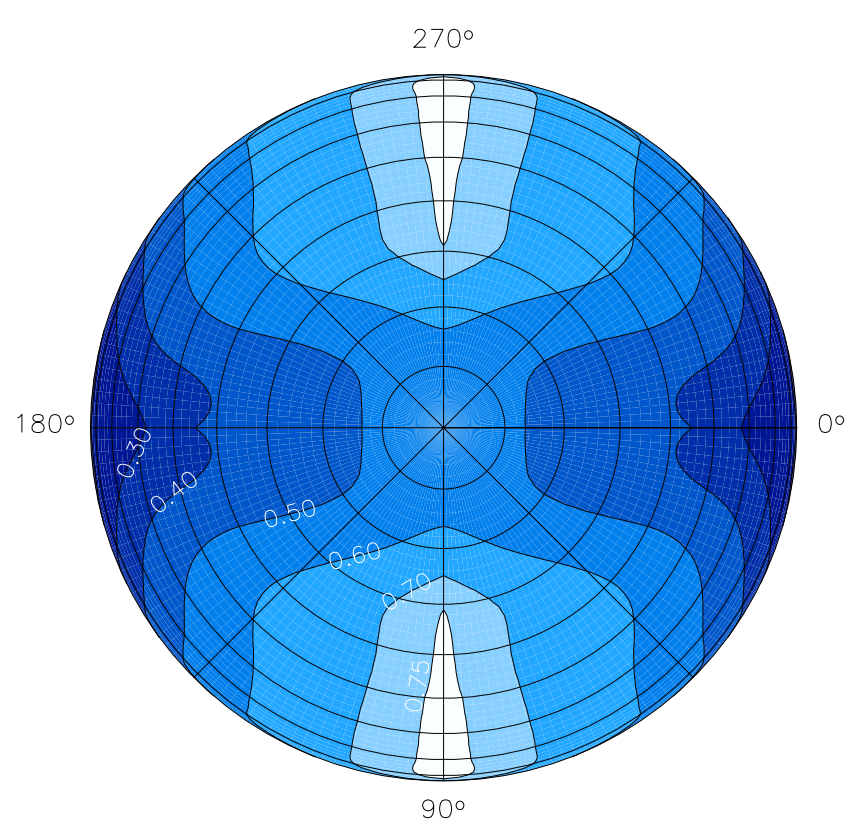

Fig. 7. Contour plot of direction weighting function $f^{\mathrm{T}}(360 \mathrm{~nm}$, based on hemispheric FEP transmittance) as a function of $\vartheta$ and $\varphi$ (orthographic projection looking from the zenith). Azimuth angles $\varphi$ are indicated $\left(0^{\circ}=\right.$ north, $180^{\circ}=$ south $)$.

In Fig. 6 calculated, angle dependent $T_{\mathrm{d}}$ for two selected wavelengths are compared with our experimental data from the FEP film actually used for the construction of the chamber walls. The agreement is satisfactory justifying the use of Eq. (21) and the parameters shown in Fig. 5 to calculate $T_{\mathrm{d}}$ and $T_{\mathrm{h}}$ in the following.

\subsubsection{Calculation of $f^{\mathrm{T}}$}

The use of $T_{\mathrm{d}}$ instead of $T_{\mathrm{h}}$ would underestimate the absolute level of transmittance and overestimate wavelength dependence (Fig. 6). On the other hand, neglecting bulk effects by using $T_{\mathrm{F}}$ would produce the opposite effects: higher transmittance and no wavelength dependence. However, so far favouring $T_{\mathrm{h}}$ for the chamber is an assumption not yet backed by experimental data. The calculations of $f^{\mathrm{T}}$ were therefore made using $T_{\mathrm{d}}, T_{\mathrm{h}}$ and $T_{\mathrm{F}}$ for comparison.

For the $\vartheta, \varphi$ combinations also used in Sect. 3.1, the $f^{\mathrm{T}}$ were calculated wavelength dependent in the range 280$420 \mathrm{~nm}$ using $5 \mathrm{~nm}$ steps (Eq. 13). Similar to the $f^{\mathrm{V}}$ also the $f^{\mathrm{T}}$ are strongly variable. As an example Fig. 7 shows a contour plot of $f^{\mathrm{T}}$ based on $T_{\mathrm{h}}$ at a wavelength of $360 \mathrm{~nm}$. Compared to the $f^{\mathrm{V}}$ (Fig. 4) the figure looks similar qualitatively but values are lower and contours are slightly shifted. The $f^{\mathrm{T}}$ range from 0.14 in few directions at $\varphi=0^{\circ}\left(180^{\circ}\right)$ and very large zenith angles to 0.77 at $\varphi=90^{\circ}\left(270^{\circ}\right)$ at mediumlarge zenith angles.

Despite this strong variability, the relative wavelength dependencies are smooth, as shown in Fig. 8 where the ratios

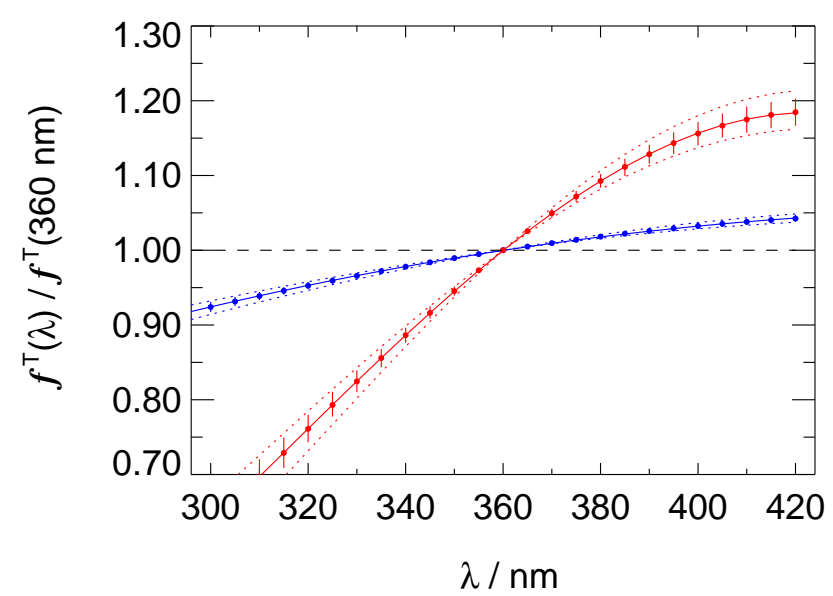

Fig. 8. Relative wavelength dependence of weighting factors $f^{\mathrm{T}}$ using a wavelength of $360 \mathrm{~nm}$ as reference. Blue: $f^{\mathrm{T}}\left(T_{\mathrm{h}}\right)$, red: $f^{\mathrm{T}}\left(T_{\mathrm{d}}\right)$. For each wavelength position the vertical bar indicates the range of results obtained within the range of zenith- and azimuth angles considered in the $f^{\mathrm{T}}$ calculations. The full lines are polynomials fitted to the averaged values. Dotted lines show relative wavelength dependencies of the corresponding transmittances of the FEP walls at angles of incidence of $0^{\circ}$ and $60^{\circ}$, respectively.

$f^{\mathrm{T}}(\lambda) / f^{\mathrm{T}}(360 \mathrm{~nm})$ are plotted. The relative wavelength dependencies closely resemble that of the corresponding transmittances of the FEP walls at medium angles of incidence also plotted in Fig. 8. Consequently, the $\vartheta, \varphi$ dependencies can be separated from the relative wavelength dependencies by selecting a reference wavelength $(360 \mathrm{~nm})$. Moreover, the ratios of $f^{\mathrm{T}}$ based on different transmittances at fixed wavelengths show little variability. At $360 \mathrm{~nm}$ the mean $f^{\mathrm{T}}$ ratios are $0.728\left(T_{\mathrm{d}} / T_{\mathrm{h}}\right)$ with a standard deviation of $1.3 \%$, and $1.124\left(T_{\mathrm{F}} / T_{\mathrm{h}}\right)$ with a standard deviation of $0.5 \%$. Thus, different FEP transmittances are producing different relative wavelength dependencies and scaling factors rather than differences in direction weighting. Therefore, unless stated otherwise, the $f^{\mathrm{T}}$ obtained at $360 \mathrm{~nm}$ assuming hemispheric FEP transmittance (Fig. 7) are used in the following. As in the case of $f^{\mathrm{V}}$ the data were parameterised by a function returning the $f^{\mathrm{T}}(\vartheta, \varphi)$ upon input of zenith- and azimuth angle.

The actual wavelength dependence of $f^{\mathrm{T}}$ will be obtained from ratios of actinic flux spectra measured simultaneously at a fixed location within and outside the chamber under overcast conditions (Sect. 4.3). In terms of the model this can be rationalised by looking at the products $s(\boldsymbol{r}, \vartheta, \varphi) T(\boldsymbol{r}, \vartheta, \varphi, \lambda)$ (Eq. 13) for single locations not too close to the chamber walls. If these products for different $\vartheta, \varphi$ are averaged, relative wavelength dependencies similar to those of Fig. 8 are obtained. Averaging is justified in the case of an isotropic radiance distribution, approximately fulfilled under overcast conditions (Sect. 3.3). Therefore, although absolute values are dependent on location, the relative 

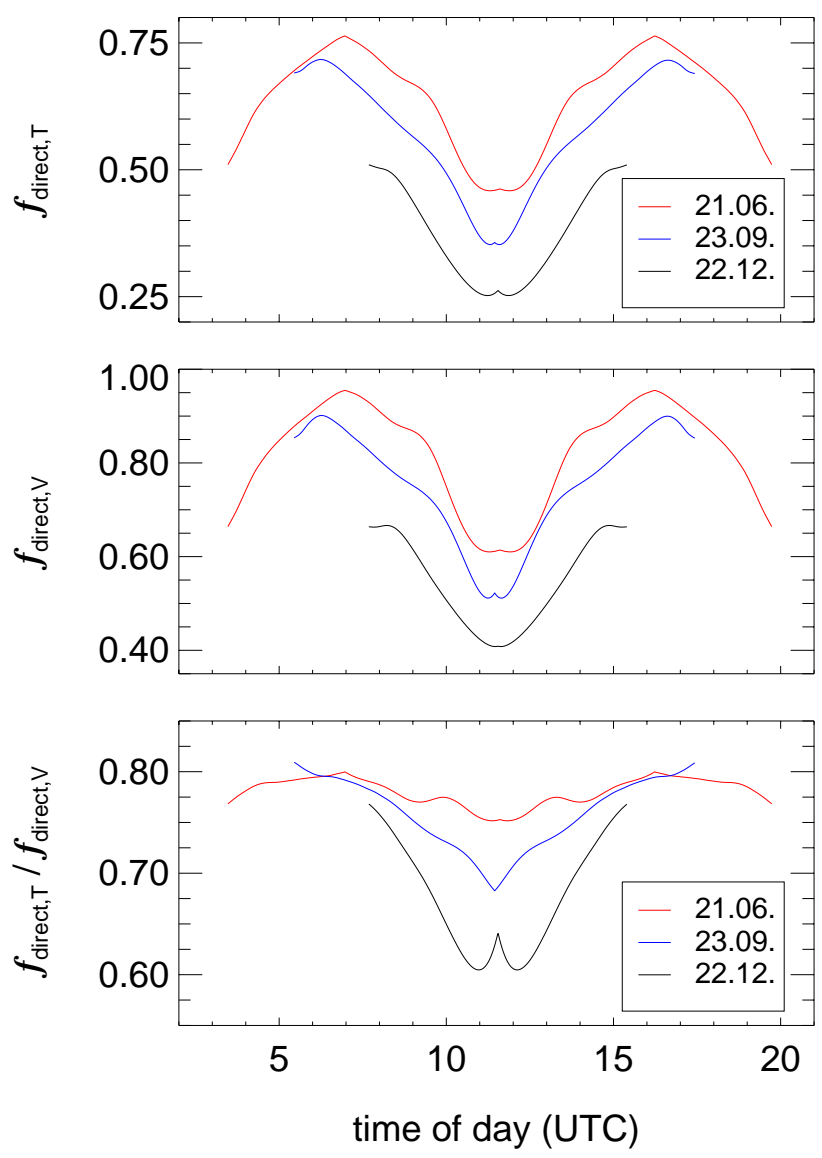

Fig. 9. Upper panels: Diurnal variations of weighting factors $f_{\text {direct, } \mathrm{T}}$ and $f_{\text {direct, } \mathrm{V}}$ on three days of the year $(\lambda=360 \mathrm{~nm})$. Lower panel: Ratio $f_{\text {direct, } \mathrm{T}} / f_{\text {direct, } \mathrm{V}}$ showing the relative importance of FEP walls transmission. Plot ranges indicate times where $\vartheta_{\circ} \leq 90^{\circ}$.

wavelength dependence of ratios of actinic flux spectra measured inside and outside under such conditions are assumed representative for the chamber.

Under clear sky conditions this type of measurements is not conclusive for the chamber as a whole because transmittances concerning direct sunlight are dependent on location and time. Moreover, model predictions for a single location are difficult to interpret because scattering processes at the chamber walls are diminishing the differences between illuminated and shaded areas. This effect is time-dependent and also affects the spectral composition of actinic flux detected locally making an interpretation difficult on the basis of the current model.

\section{$3.3 f_{\text {direct }}$ and $f_{\text {diffuse }}$}

Figure 9 gives an example of diurnal variations of $f_{\text {direct,T }}$ on three distinct days of the year. The data were obtained by inserting solar zenith- and azimuth angles into the $f^{\mathrm{T}}$ parametrisation (Eq. 15). For comparison also the illu- minated volume fractions $f_{\text {direct, } \mathrm{V}}$ (Eq. 14) and the ratios $f_{\text {direct, } \mathrm{T}} / f_{\text {direct, } \mathrm{V}}$ are plotted to demonstrate the relative contributions of shadows and FEP wall transmittance. Obviously, the fraction of the chamber illuminated by direct sunlight is highly variable with a daily minimum at noon caused by the orientation of the chamber. The effect of shadows is dominating the time dependence for direct light. However, inclusion of wall transmittance leads to additional timedependent effects which tend to amplify the impact of the shadows, particularly at winter time.

Calculation of $f_{\text {diffuse,T }}$ (Eq. 18 ) is complicated by the unknown distribution of radiance $L(\vartheta, \varphi, t)$ of diffuse sky radiation. Under overcast conditions the assumption of an isotropic distribution is simplest, i.e. $L=$ constant. Such a distribution is usually denoted UOC (uniform overcast sky). Grant and Heisler (1997) measured radiance distributions at overcast conditions in the UV range. Their data are in accord with a cosine dependence on zenith angle exhibiting little wavelength dependence:

$L(\vartheta) \propto 1+a \cos (\vartheta)$

This distribution is denoted SOC (standard overcast sky). Compared to the UOC distribution the SOC distribution is brighter at the zenith and darker at the horizon dependent on the parameter $a$. Here we use $a=1.23$ in accordance with the results of Grant and Heisler (1997). This produces radiance ratios $\mathrm{SOC} / \mathrm{UOC}$ of 1.38 at $\vartheta=0^{\circ}$ and 0.62 at $\vartheta=90^{\circ}$, respectively. At a zenith angle of $60^{\circ} \mathrm{UOC}$ and SOC radiances are similar independent of $a$.

With UOC and SOC radiance distributions weighting factors $f_{\text {diffuse }}$ are independent of time. They were calculated by numerical integrations according to Eq. (18). Different resolutions were tested for these sky scans. The use of $P=90$ corresponding to a solid angle resolution of $3 \times 10^{-4} \mathrm{sr}\left(1^{\circ} \times 1^{\circ}\right.$ at $\vartheta=90^{\circ}, \approx 21000$ sky positions) was found to be sufficiently precise, i.e. within $0.3 \%$ compared to a calculation using $P=720\left(\approx 10^{6}\right.$ sky positions). The resulting $f_{\text {diffuse }, \mathrm{V}}$ are 0.711 and 0.718 for the UOC and SOC distributions, respectively. For $f_{\text {diffuse, } \mathrm{T}}$ the corresponding values are 0.536 (UOC) and 0.544 (SOC) for a wavelength of $360 \mathrm{~nm}$. Apparently, under overcast conditions the different radiance distributions produce little differences.

Under clear sky conditions the UOC and SOC assumptions are inadequate. With the available equipment a measurement of actual radiance distributions was not feasible. Instead, analytical expressions of radiance distributions by Grant et al. (1997) were used. These expressions are based on experimental data obtained within wavelength ranges UV-A (320 $400 \mathrm{~nm})$ and UV-B (300-320 nm) dependent on zenith angle and time-dependent scattering angle $\psi$ (angular separation with respect to the sun). Calculations of $f_{\text {diffuse, }} \mathrm{V}$ and $f_{\text {diffuse, } \mathrm{T}}$ for clear sky conditions were made with the same resolution as above for the UOC and SOC distributions. In Fig. 10 examples are shown for the same days as in Fig. 9. Compared to $f_{\text {direct,T }}$ the $f_{\text {diffuse, } \mathrm{T}}$ based on the clear sky 

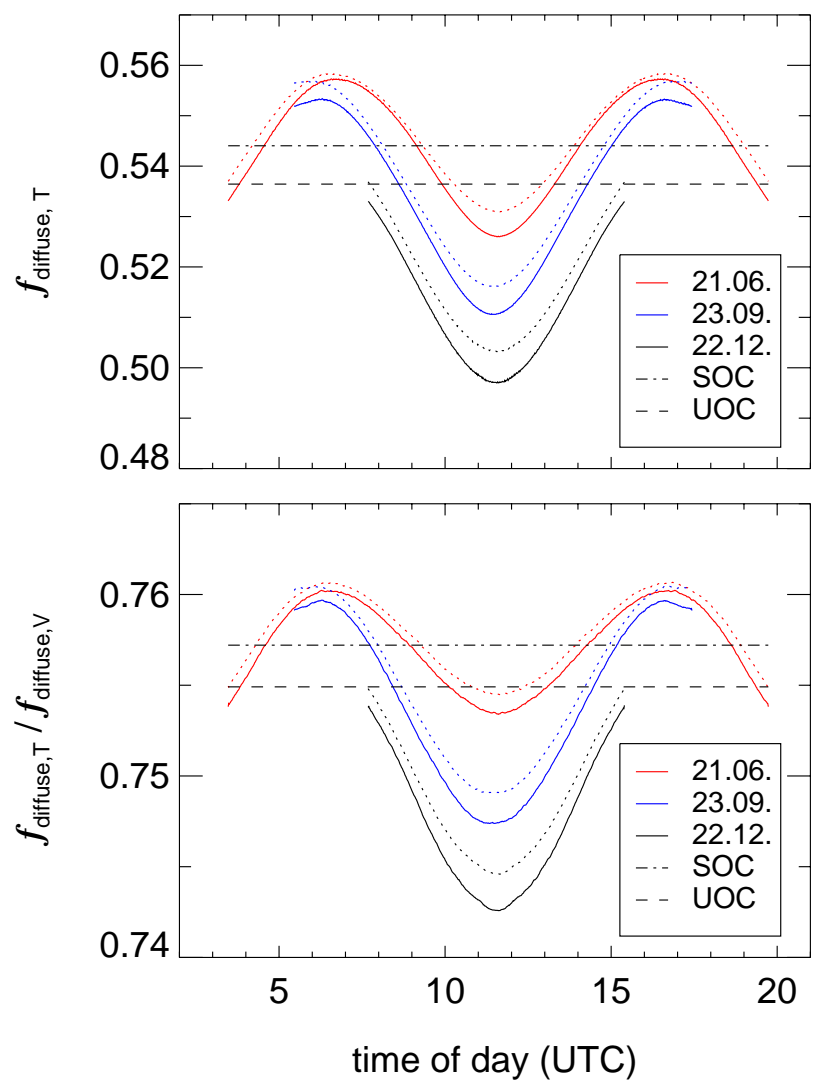

Fig. 10. Upper panel: Diurnal variations of weighting factors $f_{\text {diffuse, } \mathrm{T}}$ on three days of the year $(\lambda=360 \mathrm{~nm})$. Full and dotted lines were obtained with UV-A and UV-B radiance distributions by Grant et al. (1997), respectively. Lower panel: Ratios $f_{\text {diffuse, } \mathrm{T}} / f_{\text {diffuse, } \mathrm{V}}$ indicating the relative importance of FEP walls transmission. In both panels dashed and dashed-dotted lines show the time-independent results for UOC and SOC radiance distributions, respectively. Plot ranges indicate times where $\vartheta_{\circ} \leq 90^{\circ}$.

radiance distributions exhibit less pronounced diurnal and seasonal dependencies. The ratios $f_{\text {diffuse, } \mathrm{T}} / f_{\text {diffuse, } \mathrm{V} \text { reveal }}$ that again the effect of the shadows is dominating the timedependence. Except from a constant factor of about 0.75 , inclusion of wall transmittance leads to minor time-dependent effects $(\leq 1.5 \%)$. UV-A and UV-B radiance distributions produce very similar results with diurnal and seasonal effects slightly less pronounced in the UV-B.

\subsection{Horizon obstruction}

In addition to the chamber itself, objects in the surrounding area obstructing the horizon (buildings and tree rows) were mapped and approximated by 44 triangular elements (facades). These objects were not included in the calculations so far because they exhibit a more complex symmetry with respect to azimuth angle. Because the distance between the chamber and the surrounding objects is relatively large
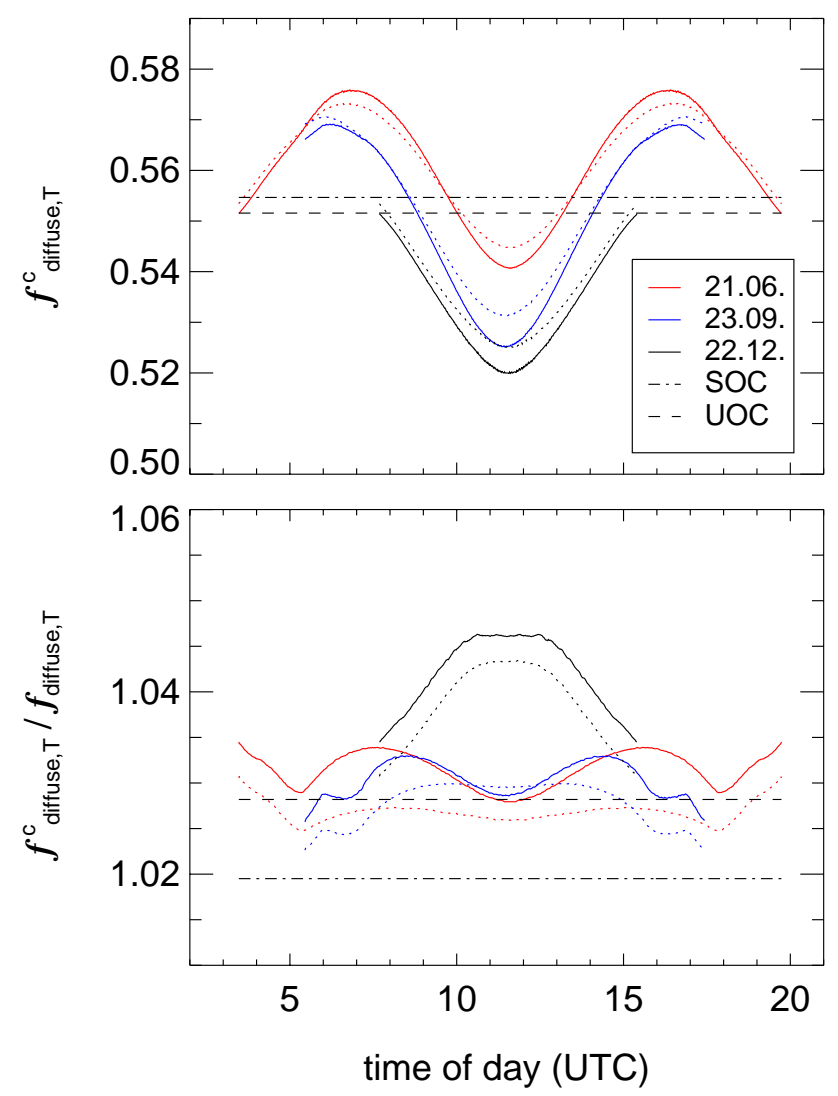

Fig. 11. Upper panel: Diurnal variations of weighting factors $f_{\text {diffuse, } T}^{\mathrm{c}}$ on three days of the year $(\lambda=360 \mathrm{~nm})$. Compared to Fig. 10 horizon obstruction was included in the calculations and y-axes are slightly shifted. Full and dotted lines were obtained with UV-A and UV-B radiance distributions by Grant et al. (1997), respectively. Lower panel: Ratios $f_{\text {diffuse, } \mathrm{T}}^{\mathrm{c}} / f_{\text {diffuse, } \mathrm{T}}$. In both panels dashed and dashed-dotted lines indicate the time-independent results for UOC and SOC radiance distributions, respectively. Plot ranges indicate times where $\vartheta_{\circ} \leq 90^{\circ}$.

compared to the chamber itself, the question whether or not an object is obstructing a direction is addressed for a single location at the centre of the chamber. The corresponding function is denoted $s_{\mathrm{c}}(\vartheta, \varphi)=\{0,1\}$. For the weighting functions of direct light this simply means multiplication by a factor 0 or 1, i.e. sunrise is delayed and sunset is premature.

$f_{\text {direct, } \mathrm{T}}^{\mathrm{c}}(t, \lambda)=f_{\text {direct, } \mathrm{T}}(t, \lambda) s_{\mathrm{c}}\left(\vartheta_{\circ}, \varphi_{\circ}\right)$

However, obstruction only occurs in some directions at zenith angles $\vartheta \geq 80^{\circ}$.

For the weighting functions of diffuse sky radiation there are two possibilities to consider horizon obstruction. Firstly, only the numerator in Eq. (18) is multiplied by $s_{\mathrm{c}}$. This yields weighting factors with respect to full view of the upper hemisphere. Secondly, the numerator and denominator in Eq. (18) are multiplied by $s_{\mathrm{c}}$ which creates weighting factors in terms of a hypothetic external measurement at the chamber-site: 

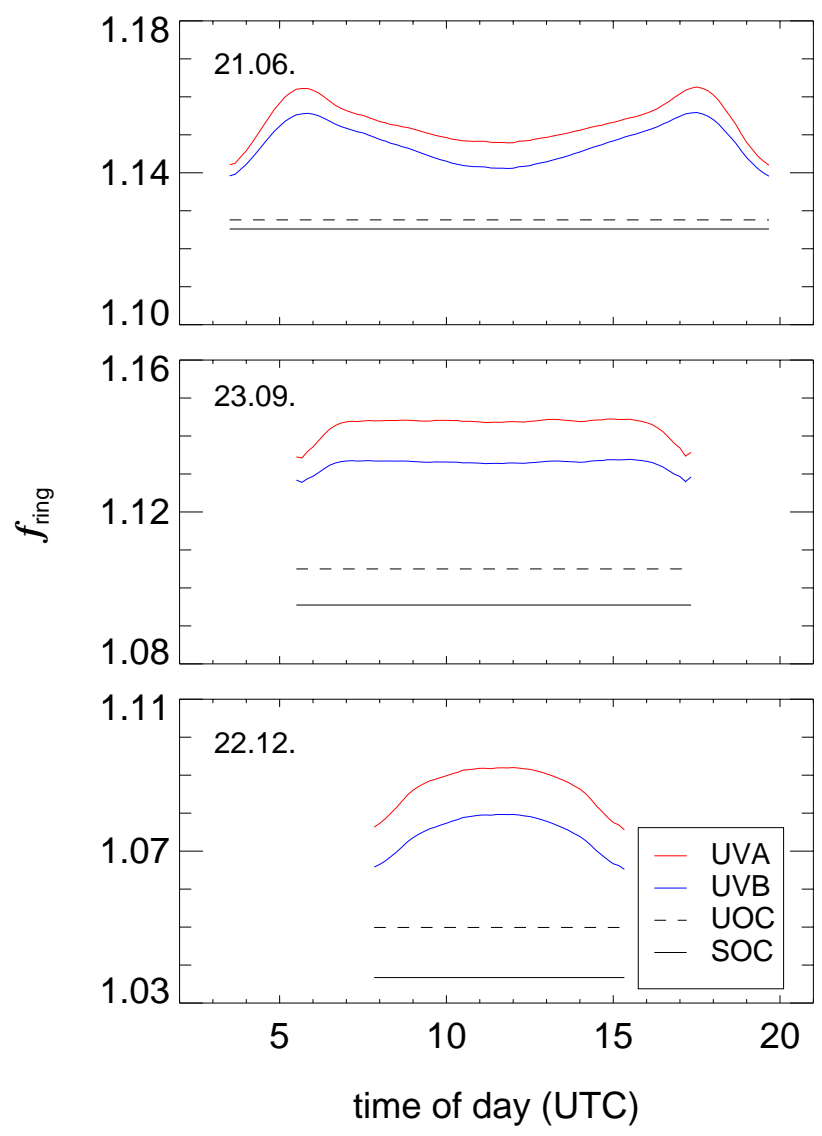

Fig. 12. Numerical ring correction factors $f_{\text {ring }}$ on three days of the year. UV-A, UV-B: clear sky radiance distributions according to Grant et al. (1997). SOC, UOC: overcast radiance distributions. Plot ranges indicates times where $\vartheta_{\circ} \leq 90^{\circ}$.

$$
\begin{aligned}
& f_{\text {diffuse, } \mathrm{T}}^{\mathrm{c}}(t, \lambda)= \\
& \frac{\sum_{p=0}^{P} \sum_{q=0}^{Q} f^{\mathrm{T}}\left(\vartheta_{p}, \varphi_{q}, \lambda\right) L\left(\vartheta_{p}, \varphi_{q}, t\right) s_{\mathrm{c}}\left(\vartheta_{p}, \varphi_{q}\right)}{\sum_{p=0}^{P} \sum_{q=0}^{Q} L\left(\vartheta_{p}, \varphi_{q}, t\right) s_{\mathrm{c}}\left(\vartheta_{p}, \varphi_{q}\right)}
\end{aligned}
$$

Regarding the integers $P$ and $Q$ the same relations apply as in Eq. (18). This second approach is more useful because also the measurement site of the spectroradiometer is subject to horizon obstruction. In Fig. 11 the $f_{\text {diffuse, } \mathrm{T}}^{\mathrm{c}}$ are shown to demonstrate the influence of the surrounding objects. Compared to Fig. 10 there is a slight change of the shape of the curves which are also shifted upwards by about 0.02 . The strongest shift is obtained at winter time.

Finally, differences between the chamber site and the site of the spectroradiometer measurements caused by horizon obstruction are accounted for by a separate function:

$$
f_{\mathrm{rc}}(t)=\frac{\sum_{p=0}^{P} \sum_{q=0}^{Q} L\left(\vartheta_{p}, \varphi_{q}, t\right) s_{\mathrm{c}}\left(\vartheta_{p}, \varphi_{q}\right)}{\sum_{p=0}^{P} \sum_{q=0}^{Q} L\left(\vartheta_{p}, \varphi_{q}, t\right) s_{\mathrm{r}}\left(\vartheta_{p}, \varphi_{q}\right)}
$$

Here $s_{\mathrm{r}}(\vartheta, \varphi)=\{0,1\}$ is addressing horizon obstruction at the site of the radiometer measurement. For $P$ and $Q$ the same relations apply as in Eq. (18). Due to the higher altitude of the spectroradiometer measurement site the $f_{\text {rc }}$ are generally slightly below unity in a range $0.97-1.0$.

Overall, horizon obstruction plays a minor role with negligible time-dependent effects.

\subsection{Shadow ring correction}

To derive $F_{\lambda}^{\text {direct }}$ and $F_{\lambda}^{\text {diffuse }}$ as a model input according to Eqs. (7) and (8), the correction factor $f_{\text {ring }}$ is needed. As described in the Experimental section, an analytical correction factor was derived considering ring geometry. However, this approach assumes an isotropic radiance distribution (UOC) and full view of the upper hemisphere.

In order to calculate ring corrections for other radiance distributions and to allow for horizon obstruction, a trianglebased model of the shadow ring with its mountings was created. Similar to the real shadow ring, the modelled ring can be tilted and shifted along an axis perpendicular to the ring plane. 200 triangles were used for a precise description of the shadow ring. Ring correction factors are obtained by performing numerical sky scans as in the previous sections (Eq. 18, 24 and 25):

$$
f_{\text {ring }}(t)=\frac{\sum_{p=0}^{P} \sum_{q=0}^{Q} s_{\text {ring }}\left(\vartheta_{p}, \varphi_{q}\right) L\left(\vartheta_{p}, \varphi_{q}, t\right)}{\sum_{p=0}^{P} \sum_{q=0}^{Q} L\left(\vartheta_{p}, \varphi_{q}, t\right)}
$$

In this equation the function $s_{\text {ring }}(\vartheta, \varphi)=\{0,1\}$ determines whether or not a ring element is blocking light coming from direction $\vartheta, \varphi$ with respect to the detector position (approximated by a single point). Concerning $P$ and $Q$ refer to Eq. (18).

In Fig. 12 examples are shown for three days of different seasons. SOC and UOC correction factors are independent of time for a given day of the year, i.e. for a fixed position of the ring. Moreover, they are not too different with a maximum difference of $1.3 \%$ during winter. At the chosen resolution of the sky scans $(P=90)$, the numerical UOC correction factors are in excellent agreement with the analytical ring corrections $(\leq 0.2 \%$ deviation).

Under clear sky conditions the $f_{\text {ring }}$ exhibit a dependence on time of day and wavelength band if the analytical radiance distributions by Grant et al. (1997) are used. Correction 
factors are somewhat higher compared to UOC and SOC distributions because the shadow ring is obstructing areas with high circumsolar radiance. This effect is slightly less pronounced in the UV-B.

In the calculations concerning ring corrections an about $0.1 \mathrm{rad}\left(\approx 6^{\circ}\right)$ scattering angle area around the sun was excluded from the sky scans. As mentioned in the Experimental section, there are practical reasons to do so. Another reason for this exclusion is that an extrapolation of the radiance distributions by Grant et al. (1997) to a position too close to the sun is not useful because the experimental data by Grant et al. (1997) were obtained at scattering angles $\psi \geq 15^{\circ}$. Calculations including the $6^{\circ}$ area around the sun give ring corrections higher by about $2.5 \%$ as a result of the strongly increasing radiance in this region, i.e. they tend to slightly increase the contribution of diffuse sky radiation (Eqs. 7 and 8). It is beyond the scope of this work to accurately distinguish direct sunlight from sky radiation. In the present approach diffuse sky radiation coming from a $6^{\circ}$-area around the sun is considered direct sunlight. This is correct in the sense that the direction where the light comes from is very close to the sun and with respect to the chamber the same corrections apply as for direct sunlight.

Ring correction factors $f_{\text {ring }}^{\mathrm{r}}$ including horizon obstruction were calculated by multiplying the numerator and the denominator of Eq. (26) by $s_{\mathrm{r}}$ (see Sect. 3.4). However, the ratios $f_{\text {ring }}^{\mathrm{r}} / f_{\text {ring }}$ are very close to unity $(0.98-1.0)$ because few directions are obstructed by both the shadow ring and surrounding objects.

\section{Solar actinic flux measurements and calculation of photolysis frequencies}

\subsection{External contributions of direct sunlight and diffuse sky radiation}

The partitioning of spectral actinic flux in direct sunlight and diffuse sky radiation is highly variable under natural conditions in particular in the presence of broken cloud fields. Under clear sky conditions the direct and diffuse contributions are expected to vary more smoothly dependent on wavelength and solar zenith angle. However, actual values also depend on other parameters such as ozone column and aerosol load which may vary in the course of a day.

As an example Fig. 13, shows actinic flux spectra and the contributions of direct and diffuse sunlight obtained under clear sky conditions on 28 July 2002 in the early morning $\left(\vartheta_{\circ}=75^{\circ}\right)$ and at local noon $\left(\vartheta_{\circ}=32^{\circ}\right)$. As expected, the contribution of direct sunlight is decreasing with decreasing wavelength and increasing solar zenith angle. A solar zenith angle of $32^{\circ}$ is close to the local minimum at summer solstice $\left(27^{\circ}\right)$ while $75^{\circ}$ is reached at local noon during winter solstice. This demonstrates the strong seasonal and diurnal
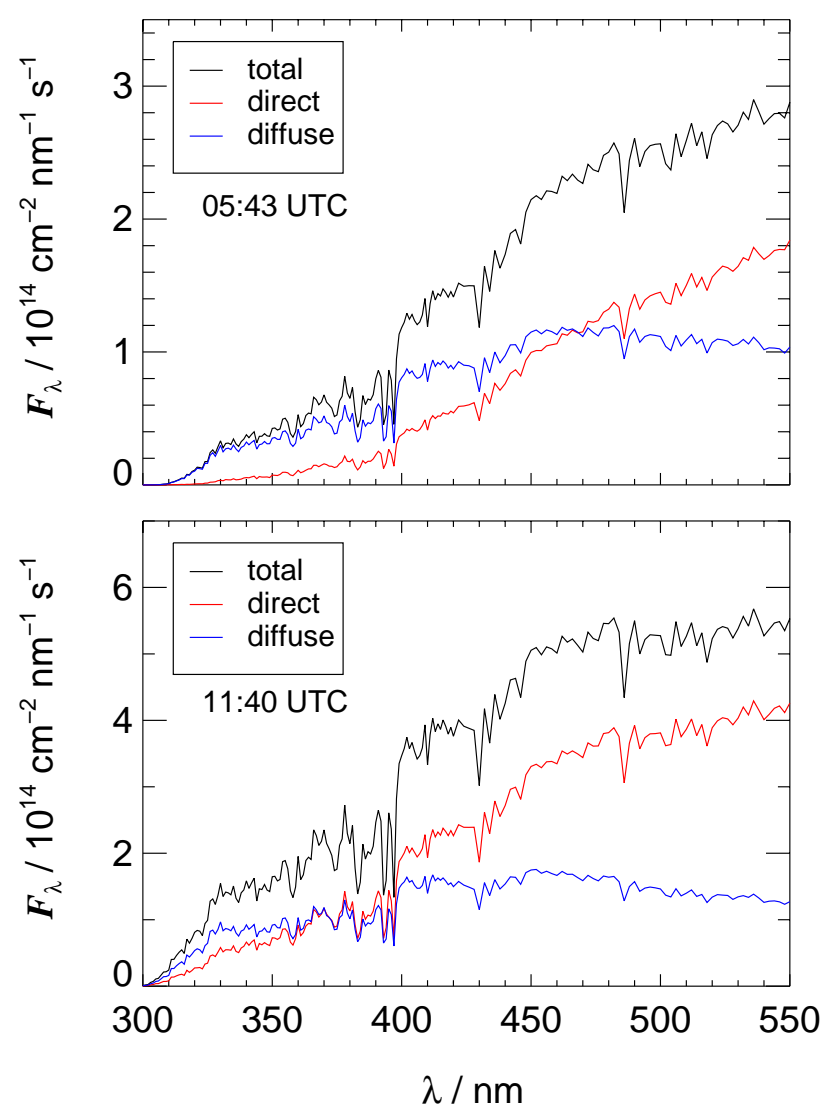

Fig. 13. Examples of solar actinic flux spectra at different times of day under clear sky conditions (28 July 2002). Upper panel: $\vartheta_{\circ}=75^{\circ}$. Lower panel: $\vartheta_{\circ}=32^{\circ}$ (local noon). The measured total actinic flux (black) is divided in direct sunlight (red) and diffuse sky radiation (blue) according to Eqs. (7) and (8).

dependence of the contributions of direct sunlight under clear sky conditions.

In Fig. 14 the diurnal variations of photolysis frequencies $j\left(\mathrm{NO}_{2}\right)$ and $j\left(\mathrm{O}^{1} \mathrm{D}\right)$ on 28 July 2002 are plotted. These data were calculated according to Eq. (6) using selected data of absorption cross sections and quantum yields for the photolysis processes (4) and (1) at $298 \mathrm{~K}$ (Merienne et al., 1995; Troe, 2000; Malicet et al., 1995; Matsumi et al., 2002). $j\left(\mathrm{O}^{1} \mathrm{D}\right)$ and $j\left(\mathrm{NO}_{2}\right)$ were chosen as examples because the corresponding photolyses are taking place in the UV-B and UV-A/VIS $(\leq 420 \mathrm{~nm})$ regions, respectively. Because of the different wavelength regimes, the relative diurnal variations as well as the contributions of diffuse and direct radiation are different for $j\left(\mathrm{NO}_{2}\right)$ and $j\left(\mathrm{O}^{1} \mathrm{D}\right)$. For example, in the case of $j\left(\mathrm{NO}_{2}\right)$ up to $50 \%$ are direct sunlight at noon. The time-dependent effects with respect to the simulation chamber are therefore expected to be more pronounced for $j\left(\mathrm{NO}_{2}\right)$ rather than for $j\left(\mathrm{O}^{1} \mathrm{D}\right)$. Other important photolysis processes (HONO, HCHO etc.) lie in between these extremes. 

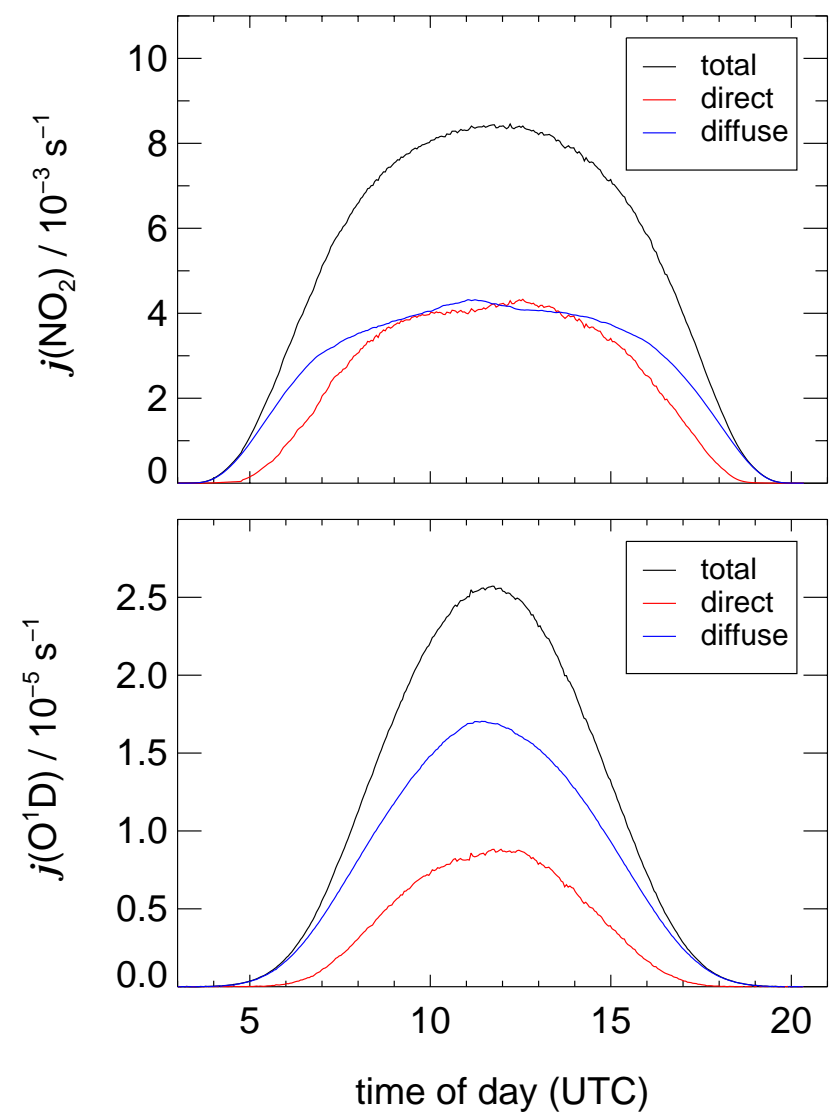

Fig. 14. Diurnal variations of $j\left(\mathrm{NO}_{2}\right)$ (upper panel) and $j\left(\mathrm{O}^{1} \mathrm{D}\right)$ (lower panel) under clear sky conditions (28 July 2002). The contributions of direct sunlight reach maxima of about $50 \%$ and $30 \%$ for $j\left(\mathrm{NO}_{2}\right)$ and $j\left(\mathrm{O}^{1} \mathrm{D}\right)$, respectively.

It should be noted that 28 July 2002 was an exceptionally clear day where the diurnal variations of total photolysis frequencies look almost perfectly symmetrical. However, the contributions of direct and diffuse radiation show slight variations which are compensating each other. Such variations are very common on clear sky days and can be attributed to changes in aerosol load.

\subsection{Experimental check of shadow ring corrections}

In Figs. 13 and 14 the contributions of diffuse and direct light were calculated using a shadow ring correction based on a UOC radiance distribution because the actual, wavelength dependent sky radiance distributions are unknown. Under overcast conditions, ring correction factors can be checked by comparison of the actinic fluxes obtained with and without shadow ring but at overcast conditions no ring correction is needed. To determine the $f_{\text {ring }}^{\mathrm{r}}$ under clear sky conditions, measurements were made where direct sunlight was occasionally blocked manually from the unshaded detector by a small disk as described in the Experimental section. Ring
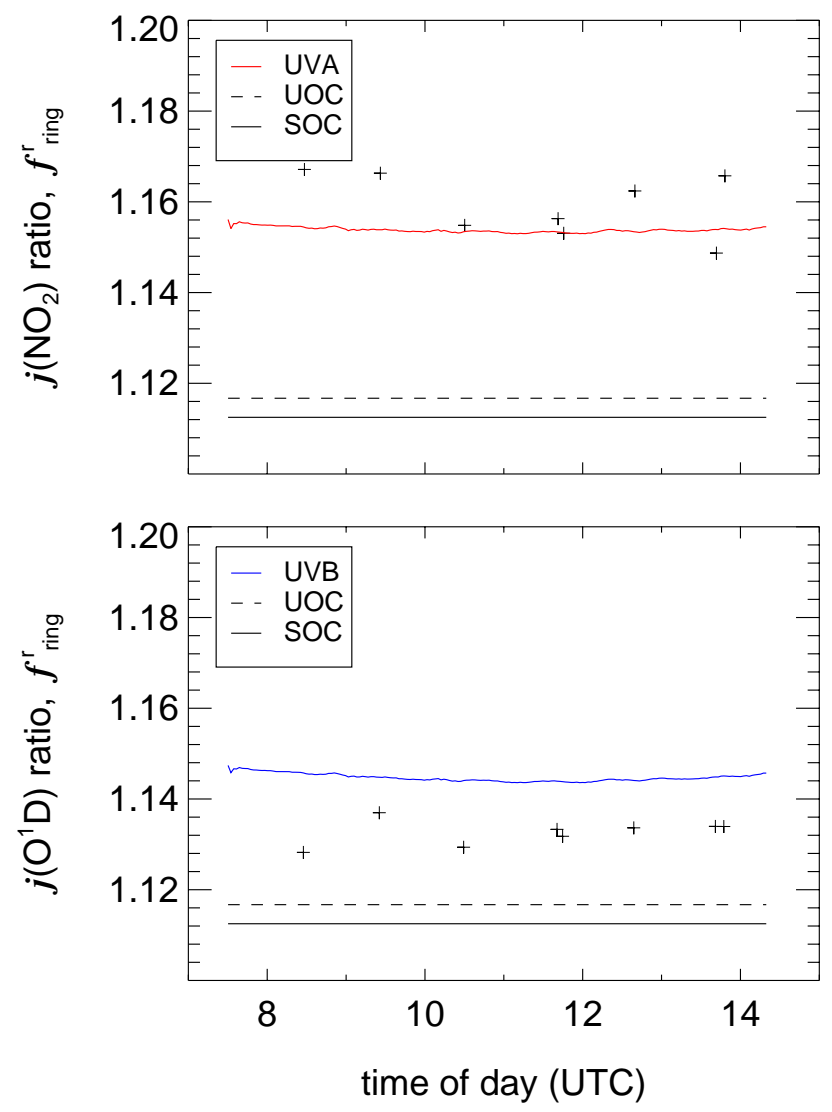

Fig. 15. Measured $j\left(\mathrm{NO}_{2}\right)$ and $j\left(\mathrm{O}^{1} \mathrm{D}\right)$ ratios obtained with detector heads obscured by a disc or by the shadow ring (crosses), and numerical shadow ring correction factors $f_{\text {ring }}^{\mathrm{r}}$ (lines) assuming different radiance distributions (17 April 2003).

correction factors are then obtained by dividing the actinic flux obtained with disc by those obtained with shadow ring. In Fig. 15 results of spot check measurements on another clear sky day (17 April 2003) are compared with the numerically calculated ring corrections for different radiance distributions. Photolysis frequencies $j\left(\mathrm{NO}_{2}\right)$ and $j\left(\mathrm{O}^{1} \mathrm{D}\right)$ are used for this comparison because they can be assigned to the spectral ranges UV-A and UV-B, respectively. Before and after these measurements the two channels of the spectroradiometer agreed within $1 \%$ which was checked by removing the shadow ring. As is evident from Fig. 15, the agreement between measured and calculated ring corrections based on distributions by Grant et al. (1997) is satisfactory for the UVA while SOC and UOC factors are too low. For the UV-B the result is not as clear. Measured ring corrections are somewhat lower than calculated for the UV-B clear sky radiance distributions but the data agree within $2 \%$. These comparisons show that, at least in the UV-A at clear sky conditions, the use of radiance distributions according to Grant et al. (1997) yields better ring corrections compared to the simpler SOC and UOC distributions. However, external parameters 
like aerosol characteristics are always influencing the actual radiance distribution under clear sky conditions. This results in unknown deviations from the analytical descriptions by Grant et al. (1997) and possibly deficient shadow ring corrections. The implications are discussed in Sect. 4.4.

\subsection{Experimental determination of the $f^{\mathrm{T}}$ wavelength de- pendence}

On two days with changing cloud cover (28-29 May 2002) measurements were made with two spectroradiometers operating simultaneously inside and outside the chamber. From the 640 spectra recorded on these two days, 150 were selected where conditions were completely overcast. The selection criterion was that the two channels outside (with/without shadow ring) received integrated actinic flux similar within $2 \%$ after shadow ring correction. As was shown in Sect. 3.2, the ratio inside/outside of actinic flux spectra under such conditions should resemble the relative wavelength dependence of the chamber as a whole, multiplied with a location dependent scaling factor. In Fig. 16 the experimental data are compared with the model calculations. A constant factor of 0.548 was factored out for this comparison to obtain a ratio of unity at $360 \mathrm{~nm}$. The experimental wavelength dependence lies between the modelled behaviour for hemispheric and direct transmittance, but closer to the hemispheric as expected. Within experimental uncertainties there is no significant curvature recognisable and consequently a straight line was fitted to the data:

$g(\lambda)=\frac{f^{\mathrm{T}}(\lambda)}{f^{\mathrm{T}}(360 \mathrm{~nm})}=1+0.00173(\lambda / \mathrm{nm}-360)$

The scaling factors calculated for the actual measurement location are $0.645\left(T_{\mathrm{F}}\right), 0.577\left(T_{\mathrm{h}}\right)$ and $0.425\left(T_{\mathrm{d}}\right)$. The calculated value of 0.577 assuming hemispheric transmittance compares best with the experimental factor of 0.548 in accordance with the relative wavelength dependence. In the following we will use $g(\lambda)$ to describe the relative wavelength dependence of $f^{\mathrm{T}}$. Any wavelength dependent influence of scattering processes at the chamber walls is also assumed to be included in $g(\lambda)$.

4.4 Calculation of actinic flux spectra and photolysis frequencies for SAPHIR

With the model tools and experimental information gathered so far, mean actinic flux spectra and photolysis frequencies for the simulation chamber can be calculated upon input of external direct and diffuse spectra, at least on a relative basis excluding the scaling factor $h^{\mathrm{c}}\left(\right.$ Eq. 9). $h^{\mathrm{c}}$ is reserved for determination by chemical actinometry (Bohn et al., 2004) which can compensate deficiencies of the model calculations with respect to internal reflections (Sect. 3). However, a reasonable attempt is to estimate $h^{\mathrm{c}}$ from $4 \pi$ sr spectroradiome-

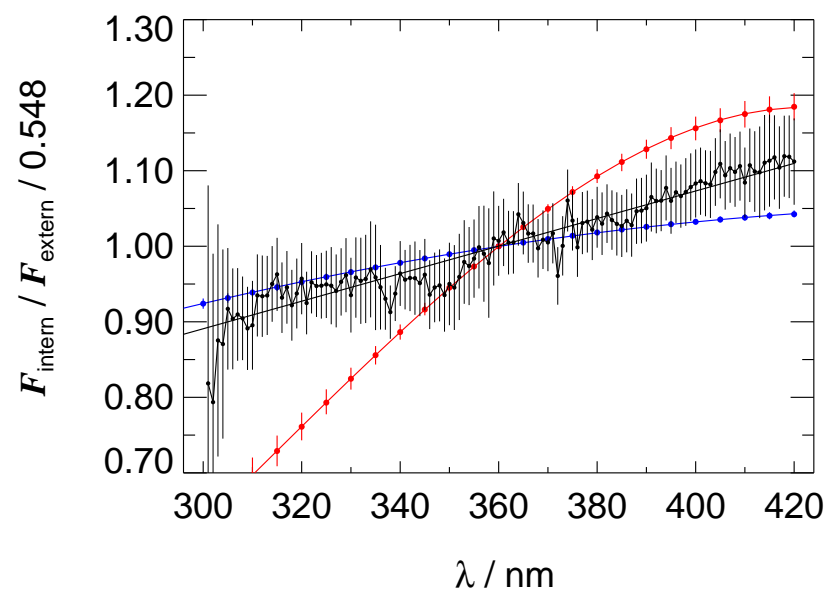

Fig. 16. Experimental ratios of solar actinic flux inside and outside the chamber and relative wavelength dependence of weighting factors $f^{\mathrm{T}}$. Black: Ratio of spectra obtained at a single location within the chamber and outside under overcast conditions (normalised to unity at $360 \mathrm{~nm}$ ). Vertical bars indicate the standard deviations of the ratios. The straight line shows a fit to the data (Eq. 27). Blue: $f^{\mathrm{T}}\left(T_{\mathrm{h}}\right)$. Red: $f^{\mathrm{T}}\left(T_{\mathrm{d}}\right)$ (see Fig. 8).

ter measurements of ratios of up- and down-welling actinic flux within the chamber:

$h^{\mathrm{c}} \approx 1+\frac{F_{\lambda}^{\uparrow}}{F_{\lambda}^{\downarrow}}$

From measurements on eight days with changing conditions $h^{\mathrm{c}} \approx 1.3$ is estimated, independent of wavelength. This preliminary factor will be used here to complete the model predictions by putting them closer to reality without anticipating the actinometric result.

Under overcast conditions the calculation of mean actinic flux spectra can be summarised as follows:

$F_{\lambda}^{\mathrm{c}}(\lambda)=h^{\mathrm{c}} g(\lambda) F_{\lambda}^{\text {diffuse }}(\lambda) f_{\text {diffuse }, \mathrm{T}}^{\mathrm{c}} f_{\mathrm{rc}}$

The time-independent factors $f_{\text {diffuse, } \mathrm{T}}^{\mathrm{c}}$ and $f_{\text {rc }}$ (Eqs. 24 and 25 ) corresponding to an SOC radiance distribution at $360 \mathrm{~nm}$ are 0.555 and 0.990 , respectively. These factors are preferred because the radiance distribution was derived from measured data (Grant and Heisler, 1997). However, UOC factors are very similar and the difference is considered insignificant within experimental uncertainties $(\approx 1 \%)$. From actinic flux spectra measured on an overcast day (6 March 2003) mean chamber spectra and photolysis frequencies were calculated resulting in typical ratios inside/outside of 0.74 and 0.67 for $j\left(\mathrm{NO}_{2}\right)$ and $j\left(\mathrm{O}^{1} \mathrm{D}\right)$, respectively. The difference is caused by the wavelength correction (Eq. 27) which also introduces a slight time-dependence for the photolysis frequencies $(\leq 1 \%)$ because the spectral distributions are changing in the course of a day.

Under clear sky conditions the procedure is more complex because direct and diffuse external actinic flux spectra have 

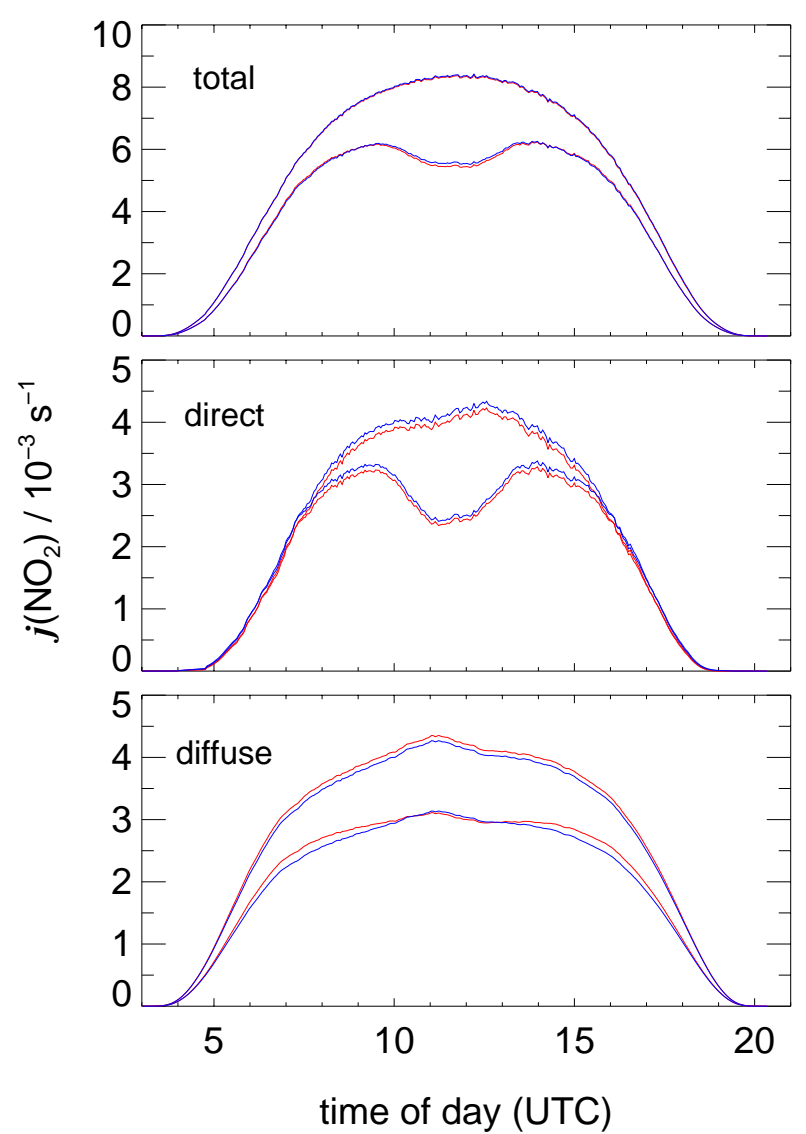

Fig. 17. Diurnal variations of $j\left(\mathrm{NO}_{2}\right)$ on a clear sky day (28 July 2002) based on different assumptions. Blue: SOC radiance distribution. Red: UV-A radiance distribution. In all panels the upper curves correspond to the external photolysis frequencies at the site of the simulation chamber. The lower curves show the photolysis frequencies inside the chamber based on the external data, modelled weighting factors and a preliminary, radiometric scaling factor $h^{\mathrm{c}}=1.3$.

to be calculated by applying shadow ring corrections $f_{\text {ring }}^{\mathrm{r}}$ (Eqs. 7 and 8). Mean actinic flux spectra are then calculated according to the following equation:

$$
\begin{aligned}
F_{\lambda}^{\mathrm{c}}(\lambda)=h^{\mathrm{c}} g(\lambda) & \left\{F_{\lambda}^{\text {diffuse }}(\lambda) f_{\text {diffuse }}^{\mathrm{c}} f_{\mathrm{rc}}\right. \\
& \left.+F_{\lambda}^{\text {direct }}(\lambda) f_{\text {direct }}^{\mathrm{c}}\right\}
\end{aligned}
$$

To investigate the differences introduced by different radiance distributions, clear-sky calculations were made based on UV-A, UV-B, SOC and UOC distributions. 28 July 2002 was selected for this comparison because the contribution of direct sunlight is at a maximum on a clear-sky summer day. $j\left(\mathrm{NO}_{2}\right)$ and $j\left(\mathrm{O}^{1} \mathrm{D}\right)$ are again used to mark the limiting behaviour also for other photolysis frequencies. As in the case of overcast conditions, differences resulting from the use of SOC and UOC distributions are small $(\leq 1 \%)$. Also the differences arising from UV-A and UV-B distributions are minor $(\leq 1 \%)$ and considered negligible. Figs. 17 and 18 are
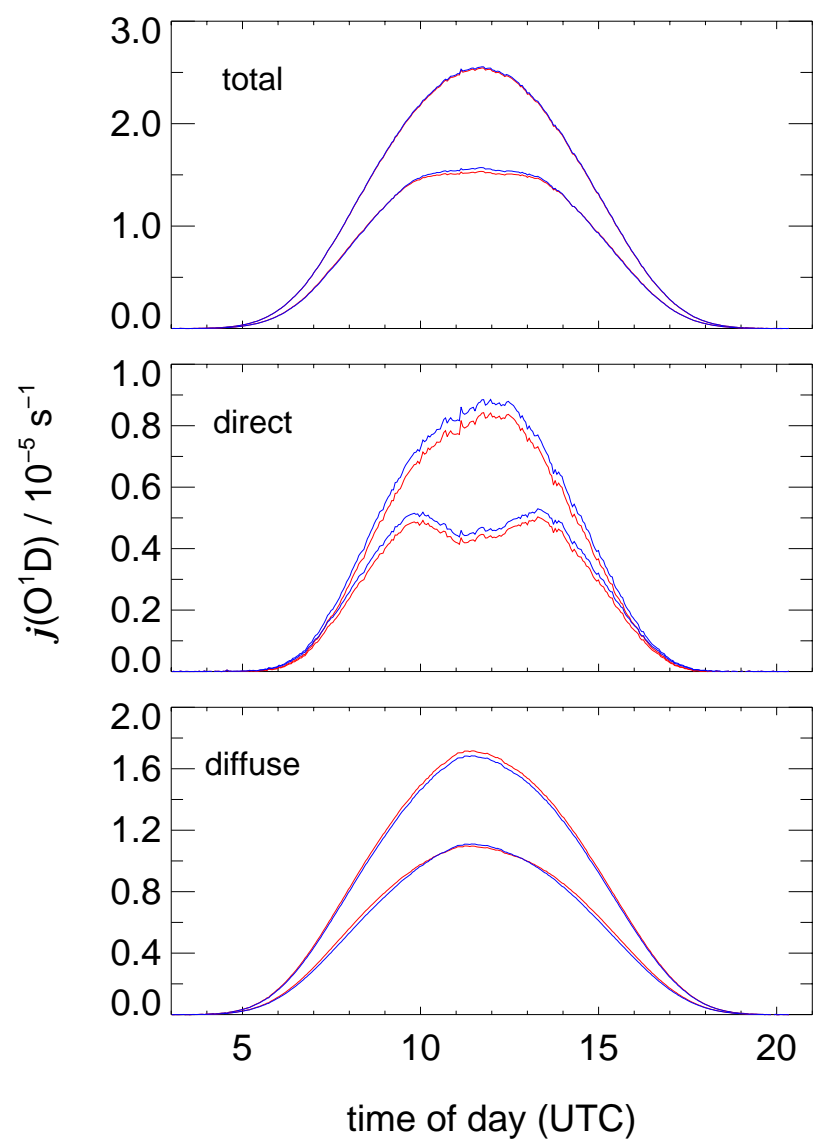

Fig. 18. Diurnal variations of $j\left(\mathrm{O}^{1} \mathrm{D}\right)$ on a clear sky day (28 July 2002). See Fig. 17 for explanation.

showing the results using SOC and UV-A radiance distributions and the contributions of diffuse and direct sunlight. The ratios inside/outside of total photolysis frequencies are strongly time-dependent under clear sky conditions. On 28 July 2002 they vary between 0.65 and 0.86 with a mean of 0.78 for $j\left(\mathrm{NO}_{2}\right)$ and between 0.60 and 0.73 with a mean of 0.68 for $j\left(\mathrm{O}^{1} \mathrm{D}\right)$. Thus, the mean ratios are comparable to overcast conditions and the time-dependence is more pronounced in the case of $j\left(\mathrm{NO}_{2}\right)$, as expected.

Apparently, the assumption of different radiance distributions has limited influence under clear sky conditions. Radiance distributions are affecting the shadow ring corrections as well as the chamber-specific factors which leads to partly compensating effects. For example, in the SOC case the contribution of diffuse sky radiation is lower, but time independent. On the other hand, the larger direct part is subject to a stronger time-dependent variation. As a consequence the $j$ ratios SOC/UV-A for the chamber are close to unity. They are merely varying between 0.990 at sunrise and 1.020 at noon for $j\left(\mathrm{NO}_{2}\right)$ and between 0.985 at sunrise and 1.025 at noon for $j\left(\mathrm{O}^{1} \mathrm{D}\right)$. Deviations of the radiance distribution from the analytical distributions by Grant et al. (1997), e.g. 
caused by the aerosol load, are therefore not expected to be a major problem.

In principle the quantity $F_{\lambda}^{\text {direct }}$ measured externally contains information on the aerosol which could be used to derive improved radiance distributions using a radiative transfer model. However, clear sky conditions are fairly uncommon at Jülich and the influence of the radiance distribution is expected to be more pronounced under broken cloud conditions or heterogeneous cloud cover. In the future, we are therefore planning to monitor relative radiance distributions by a UV sensitive sky imager to create additional input for the model, preferably in various wavelength bands. This approach should be feasible independent of external conditions. However, presently the problem of unknown radiance distributions is not solved and it is hard to estimate the errors possibly introduced by clouds under unfavourable conditions.

\section{Conclusions}

In this work mean photolysis frequencies for a sunlit, nonhemispheric atmosphere simulation chamber with opaque construction elements were derived based on external measurements of diffuse and direct solar actinic flux and model calculations. Time-dependent effects are most pronounced for direct sunlight (up to a factor of two, Fig. 9). Both diurnal and seasonal effects are of importance. The influence of the FEP walls, of different distributions of diffuse sky radiation and of horizon obstruction were found to be of minor importance but the impact of clouds needs further investigation. The distinction between direct and diffuse actinic flux by using a simple shadow ring for the external measurement was found to be sufficiently precise for the purpose of this work.

Photolysis frequencies within the simulation chamber are lower than outside by typically $20-30 \%$. Given the high natural variability of photolysis frequencies under tropospheric conditions this reduction is not significant for the research objective of SAPHIR, i.e. the study of tropospheric chemistry under natural conditions.

To investigate the quality of the predictions the calculated photolysis frequencies were compared with data from chemical actinometry within the chamber. The validity of the model assumptions is confirmed by a linear correlation of the data, independent of external conditions and season. More details concerning this comparison are given by Bohn et al. (2004).

The influence of scattering processes at the chamber walls and of reflections within the chamber will be investigated by an extended model based on more detailed information concerning FEP film scattering properties.

The method described in this work can also be used to predict lighting conditions in other complex environments e.g. in the vicinity of buildings or vegetation. Such information is necessary to model local photochemistry, but also, for ex- ample, to assess the exposure of humans to UV radiation as a function of time and external conditions.

Acknowledgements. The authors thank H. Schütze (Plantec GmbH) for providing SAPHIR CAD data, D. Bartel (FZ-Jülich) for his help with AUTOCAD, G. M. Wallner (University of Leoben, Austria) for providing data on the spectral transmittance of FEP film and F. Rohrer and T. Brauers for useful discussions. Financial support by the Helmholtz-Gemeinschaft (B.B.) is gratefully acknowledged.

Edited by: U. Pöschl

\section{References}

Bohn, B., Rohrer, F., Brauers, T., and Wahner, A.: Actinometric measurements of $\mathrm{NO}_{2}$ photolysis frequencies in the atmosphere simulation chamber SAPHIR, Atmos. Chem. Phys. Discuss., 4, 8141-8170, 2004,

SRef-ID: 1680-7375/acpd/2004-4-8141.

Grant, R. H. and Heisler, G. M.: Obscured overcast sky radiance distributions for ultraviolet and photosynthetically active radiation, J. Appl. Meteorol., 36, 1336-1345, 1997.

Grant, R. H., Heisler, G. M., and Gao, W.: Clear sky radiance distributions in ultraviolet wavelength bands, Theo. Appl. Climatol., 56, 123-135, 1997.

Harrison, L., Michalsky, J., and Berndt, J.: Automated multifilter rotating shadow-band radiometer: An instrument for optical depth and radiation measurements, Appl. Opt., 33, 5118-5125, 1994.

Hofzumahaus, A., Kraus, A., and Müller, M.: Solar actinic flux spectroradiometry: A technique for measuring photolysis frequencies in the atmosphere, Appl. Opt., 38, 4443-4460, 1999.

Kraus, A. and Hofzumahaus, A.: Field measurements of atmospheric photolysis frequencies for $\mathrm{O}_{3}, \mathrm{NO}_{2}, \mathrm{HCHO}, \mathrm{H}_{2} \mathrm{O}_{2}$ and HONO by UV spectroradiometry, J. Atmos. Chem., 31, 161180, 1998.

Malicet, J., Daumont, D., Charbonnier, J., Parisse, C., Chakir, A., and Brion, J.: Ozone UV Spectroscopy II: Absorption crosssections and temperature dependence, J. Atmos. Chem., 21, 263 273, 1995.

Matsumi, Y., Comes, F. J., Hancock, G., Hofzumahaus, A., Hynes, A. J., Kawasaki, M., and Ravishankara, A. R.: Quantum yields for production of $\mathrm{O}\left({ }^{1} \mathrm{D}\right)$ in the ultraviolet photolysis of ozone: Recommendation based on evaluation of laboratory data, J. Geophys. Res., 107, D3, doi:10.1029/2001JD000510, 2002.

Merienne, M. F., Jenouvrier, A., and Coquart, B.: The $\mathrm{NO}_{2}$ absorption spectrum: 1. Absorption cross-sections at ambient temperature in the $300-500 \mathrm{~nm}$ region, J. Atmos. Chem., 20, 281-297, 1995.

Müller, M., Kraus, A., and Hofzumahaus, A.: $\mathrm{O}_{3} \rightarrow \mathrm{O}\left({ }^{1} \mathrm{D}\right)$ photolysis frequencies determined from spectroradiometric measurements of solar actinic UV-radiation: Comparison with chemical actinometer measurements, Geophys. Res. Lett., 22, 679-682, 1995.

Rohrer, F., Bohn, B., Brauers, T., Brüning, D., Johnen, F.-J., Wahner, A. and Kleffmann, J.: Characterisation of the photolytic HONO source in the atmosphere simulation chamber SAPHIR, Atmos. Chem. Phys. Discuss., 4, 7881-7915, 2004,

SRef-ID: 1680-7375/acpd/2004-4-7881. 
Shetter, R. E. and Müller, M.: Photolysis frequency measurements using actinic flux spectroradiometry during PEM-Tropics Mission: Instrumentation description and some results, J. Geophys. Res., 104, 5647-5661, 1999.

Teichert, C., Haas, A., Wallner, G. M., and Lang, R. W.: Nanometer scale characterisation of polymer films by atomic-force microscopy, Macromol. Symp., 181, 457-466, 2002.
Troe, J.: Are primary quantum yields of $\mathrm{NO}_{2}$ photolysis at $\lambda \leq 398$ nm smaller than unity?, Z. Phys. Chem., 214, 573-581, 2000.

Wallner, G. M.: Kunststoffe für die transparente Wärmedämmung Polymerphysikalische Einfüsse und Modellierung, PhD-Thesis, University of Leoben, 2000.

Wallner, G. M., Lang, R. W., Platzer, W., and Teichert, C.: Optical properties of polymer films for transparent insulation, Macromol. Symp., 181, 399-409, 2002. 